\title{
Large-Eddy Simulation of Dispersion from Line Sources in a Turbulent Channel Flow
}

\author{
V. B. L. Boppana · Z-T Xie · I. P. Castro
}

Received: date / Accepted: date

\begin{abstract}
Large-eddy simulations of the dispersion from scalar line sources at various locations within a fully developed turbulent channel flow at $R e=u h / v=10400$ are presented. Both mean and fluctuating scalar quantities are compared with those from the single available set of experimental data (Lavertu and Mydlarski, 2005) and differences are highlighted and discussed. The results are also discussed in the context of scalar dispersion in other kinds of turbulent flows, e.g. homogeneous shear-flow. Initial computations at a much lower Reynolds number are also reported and compared with the two available direct numerical simulation data sets.
\end{abstract}

Keywords Channel flow $\cdot$ Dispersion $\cdot$ Line source $\cdot$ Source size

\section{Introduction}

It is of great importance to be able to predict dispersion in high Reynolds-number flows, e.g. pollutant dispersion from and within streets in urban environments. Large-eddy simulation (LES) is a promising numerical approach for this purpose in that it has already been shown to perform well for determining the mean flow and turbulence characteristics of such flows (e.g. Xie and Castro, 2008). Before studying typical street-scale dispersion using LES (with results discussed in Xie and Castro, 2009; Boppana et al., 2010) we addressed the conceptually simpler case of scalar dispersion in a channel flow, largely because there are some existent laboratory experiments and direct numerical simulations (DNS) for such flows; it is this topic that is discussed in the present paper.

Scalar dispersion in turbulent shear flows is a classical problem that has been studied for a long time in several ways. In order to understand the mixing and transport processes of scalars in the kind of turbulent flows that are prevalent in many engineering applications, experiments and numerical computations have been conducted, and theories and models developed. Some of these studies have considered point sources and others have concentrated

V. B. L. Boppana · Zheng-Tong Xie · Ian P. Castro

School of Engineering Sciences

University of Southampton

E-mail: v.b.boppana@soton.ac.uk 
on line or area sources; the scalars have almost always been considered passive for simplicity. The general problem is quite challenging for numerous reasons. To mention two: unlike the velocity field, there is strong coupling between the large and small scales in the scalar field, which doesn't admit universality concepts, and the energy spectra of velocity and scalar fluctuations can differ significantly (Warhaft, 2000). There is a considerable literature on dispersion in various turbulent fields, including homogeneous isotropic turbulence and both homogeneous and inhomogeneous turbulent shear flows. Excellent reviews on some of these are given by Sreenivasan (1991), Warhaft (2000), Shraiman and Siggia (2000) and Lavertu and Mydlarski (2005). A brief overview is given below, by way of introduction to the specific situation considered in the present paper.

One of the earliest experimental studies on the most fundamental problem of line source dispersion in grid turbulence was by Townsend (1954), for grid Reynolds numbers from 2700 to 21,000. Warhaft (1984) extended this to cases in which there was more than one line source, considering the interference between two or more line source wakes at a grid Reynolds number of $1.09 \times 10^{4}$. These studies shed light on the distribution of the mean scalar concentration downstream of the source, the rate of spread of the scalar wake and the correlation coefficients of the scalar fluctuations produced by more than one source. Similarities and differences in the character of line source dispersion in uniform shear flows and homogeneous isotropic turbulent flows were identified by Karnik and Tavoularis (1989). They stated that a universal turbulent diffusivity tensor could be used for rough estimates of both second and third-order temperature-velocity correlations. The implication is that the gradient transport concept can be used at least qualitatively to interpret the variation of all measured temperature-velocity correlations. They also explained the role played by the relative size of energy-containing eddies with respect to the wake width in the appearance and suppression of double peaks in the scalar fluctuation profiles.

Experiments in inhomogeneous flows are more limited, but include investigation of dispersion from a line source in a channel flow (Lavertu and Mydlarski, 2005) and a point source in a boundary layer flow (Fackrell and Robins, 1982). The latter conducted experiments with both ground-level and elevated sources. They studied in considerable detail the effects of source size on the scalar fluctuations and the relative importance of the terms (e.g. advection, production, diffusion and dissipation) in the variance transport equation. Lavertu and Mydlarski (2005) used a nominally two dimensional fully developed turbulent channel flow at two different Reynolds number, $R e=10,400$ and 22,800 (based on channel halfwidth, $h$, and the mean velocity at the channel centre, $U$ ). Scalar mixing from line sources placed at three different wall-normal locations was studied. Various statistical quantities of the scalar wake, e.g. the mean and fluctuation profiles, probability density functions (PDF) of the fluctuations, and second and third order velocity-scalar correlations, were obtained and compared for different source locations and at the two Reynolds numbers. In the present paper, LES results for the $R e=10400$ case are compared with the experimental data.

In the modelling context, PDF models have been developed for scalar mixing in turbulent flow. Anand and Pope (1985), for example, studied the thermal wake behind a line source in grid turbulence using a velocity/temperature joint PDF transport equation and compared results with the experimental data of Warhaft (1984). With an unconditional PDF, they successfully modelled the mean temperature field but not the variance. Using a joint PDF conditional on the lateral velocity, however, the normalised variance profiles were within a factor of two of the experimental profiles. A three-dimensional stochastic model for particle pair motion in isotropic, high Reynolds number turbulent flow was presented by Thomson (1990), who found the resulting normalised scalar variance to be in agreement with the experimental data of Fackrell and Robins (1982). Bakosi et al. (2007) developed the IECM 
(Interaction by Exchange with the Conditional Mean) model to obtain the joint PDF of the velocity and scalar. The first and second moments of the scalar from two line sources at $y_{s} / h=0.067$ and 1 in a high-aspect-ratio channel flow at a Reynolds number (based on $h$ and the friction velocity $u_{\tau}$ ) $R e_{\tau}=1080$ were compared with the experiments of Lavertu and Mydlarski (2005). Comments on these comparisons will be made later (in §4.2.1, 4.2.2 and 4.2.3). Viswanathan and Pope (2008) incorporated the effects of molecular diffusion in their modified IECM mixing model to study dispersion from line sources in grid turbulence. Some of the key predictions of the model are that the source size effects are limited to an initial period and at large times the relative intensity of the scalar fluctuations tends to 0.4 , independent of Reynolds number. The conventional Reynolds averaged Navier-Stokes (RANS) models for scalar dispersion in the regions where the convection is dominant, tend not to be as successful as models like those mentioned above.

There have been some limited direct numerical simulation (DNS) studies of point source dispersion. For example, a study of point sources in a fully developed pipe flow at $R e=$ 2650 was performed by Brethouwer et al. (1999), who also obtained satisfactory agreement with the experiments of Brown and Bilger (1996) on scalar mixing in grid turbulence. A stochastic model was tested by Iliopoulos and Hanratty (1999) using the data obtained from their DNS study on point source dispersion in fully developed channel flow at $R e=4520$. Bernard and Rovelstad (1994) examined the accuracy of random flight and closure models in predicting the turbulent scalar transport rates. Their scalar fields were produced by uniform (area) and line sources in a channel flow at $R e_{\tau}=125$. Mixing and reaction processes in an active scalar wake from a line source in channel flow (at $R e_{\tau}=180$ ) were studied by Brethouwer and Nieuwstadt (2001) in order to investigate the closure terms that appear in PDF and Conditional Moment Closure models. Scalar dispersion and mixing from two line sources in a fully developed channel flow at $R e_{\tau}=180$ was simulated by Vrieling and Nieuwstadt (2003). They formulated a new model to describe the plume dispersion and the model results seem to agree qualitatively with their DNS data. Another recent DNS study for channel flow at $R e_{\tau}=180$ (Fabregat et al., 2009) considered both a neutrally buoyant scalar, mixed convection and a buoyant scalar. Finally, in the context of grid turbulence, Livescu et al. (2000) used DNS data to study the structure and development of the scalar wake in grid turbulence, varying the sizes of the line source from the Kolmogorov microscale to an integral scale. Their scalar computations mainly simulated the turbulent convective regime and the transition to the turbulent diffusive regime, and hence the DNS data disagreed with the gradient diffusion model. This is because the latter is valid only in the full turbulent diffusion stage.

As mentioned above, some of these DNS studies were conducted in order to test typical assumptions used in closure models. Other studies that focussed on modelling the thermal dispersion from a line source are those of Kyong and Chung (1987), who incorporated a composite time scale in the gradient transport model for a homogeneous shear flow, Cho and Chung (1997), who used a second order Reynolds stress / heat flux closure model for uniform shear flow, and Wang and Komori (1999) who compared a second moment closure model with an algebraic stress and flux model for dispersion in a turbulent boundary layer.

DNS is obviously not a currently viable approach for simulating scalar dispersion in urban environments but there is some hope that LES could be a useful tool. There have been some earlier studies that used LES methods - for a point source in a street canyon (Walton and Cheng, 2002), a line source in a street canyon (Liu and Barth, 2002) and ground-level and elevated point sources in turbulent boundary layers (Sykes and Henn, 1992), for example. Also, Xie et al. (2004) have predicted possible extreme concentrations from groundlevel and elevated point sources in boundary layers. However, scalar dispersion even in 
classical flows like plane channels or turbulent boundary layers at high $R e$ is a challenging task due not least to the requirement of fine resolution, the existence of high intermittencies and the presence of steep scalar gradients. So as an initial test of the LES approach, a conceptually fundamental problem was addressed: that of dispersion from a line source in a fully developed channel flow at relatively high $R e$ number; this should be even simpler than predicting dispersion from a point source in turbulent boundary layers, e.g. Xie et al. (2004). The former is essentially a two-dimensional problem with well-defined upper and bottom boundary conditions in an axially homogeneous flow, whereas the latter is a three-dimensional problem usually with an artificially designed upper boundary condition.

In the work described here, an initial validation study was done for $R e_{\tau}=180$, with results compared with the previous DNS studies in literature. This is discussed in $\S 3$ after details of the numerical methods are presented in $\S 2$. The major part of the work - simulations of the experiments of Lavertu and Mydlarski (2005) (thermal dispersion from three line sources in a fully developed channel flow at $R e_{\tau}=520$ ) - is contained in $\S 4$. This includes consideration of the mean and fluctuating scalar fields, along with eddy diffusivities which would be difficult to obtain from laboratory experiments. Conclusions are drawn in $\S 5$.

\section{Numerical details and Settings}

2.1 Flow equations and boundary conditions

The filtered continuity and Navier-Stokes equations governing unsteady incompressible flow are

$$
\text { and } \begin{aligned}
\frac{\partial u_{i}}{\partial x_{i}} & =0 \\
\frac{\partial u_{i}}{\partial t}+\frac{\partial u_{i} u_{j}}{\partial x_{j}} & =-\frac{1}{\rho} \frac{\partial p}{\partial x_{i}}+\frac{1}{\rho} \frac{\partial}{\partial x_{j}}\left(\tau_{i j}+\mu \frac{\partial u_{i}}{\partial x_{j}}\right) .
\end{aligned}
$$

The resolved-scale velocity and pressure are respectively given by $u_{i}$ and $p$ with $u_{1}=u$, $u_{2}=v$ and $u_{3}=w$ the streamwise, vertical and lateral velocity components respectively. $\rho$ and $\mu$ are the density and dynamic viscosity of the fluid. $\tau_{i j}$ is the subgrid-scale (SGS) Reynolds stress and was handled using the Smagorinsky model in conjunction with a Lilly damping function near the walls. Smagorinsky's constant $C_{S}$ was chosen as 0.065 (Shah, 1998). In the streamwise $(x)$ and lateral $(z)$ directions, periodic boundary conditions were employed. No slip conditions were set on the channel walls $(y=0$ and $2 h$, where $h$ is half the channel height).

\subsection{Scalar equation and boundary conditions}

The filtered governing equation of the scalar is

$$
\frac{\partial c}{\partial t}+\frac{\partial u_{j} c}{\partial x_{j}}=\frac{\partial}{\partial x_{j}}\left(\left(k_{s}+k_{m}\right) \frac{\partial c}{\partial x_{j}}\right)+S
$$

where $c$ is the resolved-scale concentration of the scalar. $k_{s}$ is the subgrid turbulent diffusivity and is given by $v_{s} / S c_{s}$ where $v_{s}$ is the subgrid viscosity and $S c_{s}$ is the subgrid Schmidt 


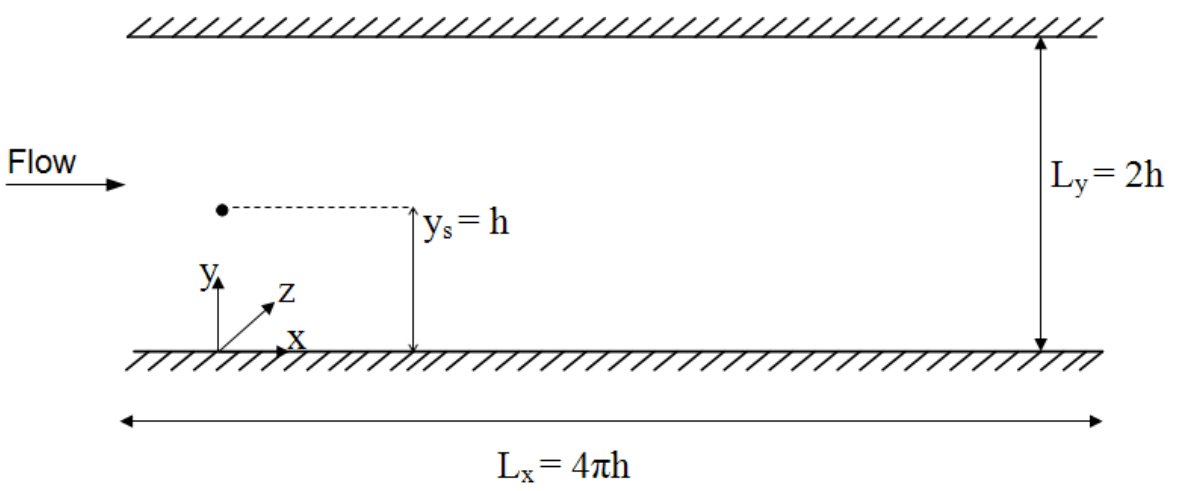

Fig. 1 A schematic representation of the central line source in the channel (not to scale).

Table 1 Details of the computational domain

\begin{tabular}{|c|c|c|c|c|c|c|}
\hline$R e_{\tau}$ & Computations type & $L_{x} \times L_{y} \times L_{z}$ & $\Delta x^{+}$ & $\Delta y_{w}^{+}$ & $\Delta y_{c}^{+}$ & $\Delta z^{+}$ \\
\hline \multirow{5}{*}{180} & LES & $4 \pi h \times 2 h \times 2 \pi h$ & 9.4 & 2.0 & 2.8 & 9.4 \\
\hline & DNS (Brethouwer and Nieuwstadt, 2001) ${ }^{\mathrm{a}}$ & $10 h \times 2 h \times 6 h$ & 8.0 & - & 4.6 & 9.0 \\
\hline & DNS (Moser et al., 1999) & $4 \pi h \times 2 h \times \frac{4}{3} \pi h$ & 17.7 & - & 4.4 & 5.9 \\
\hline & DNS (Fabregat et al., 2009) & $8 \pi h \times 2 h \times 2 \pi h$ & 17.7 & 0.4 & 4.8 & 8.8 \\
\hline & DNS (Vrieling and Nieuwstadt, 2003) ${ }^{\mathrm{a}}$ & $10 h \times 2 h \times 6 h$ & 12.0 & - & 2.4 & 11.2 \\
\hline 520 & LES & $4 \pi h \times 2 h \times \pi h$ & 27.2 & 2.0 & 16.6 & 13.6 \\
\hline 590 & DNS (Moser et al., 1999) & $2 \pi h \times 2 h \times \pi h$ & 9.7 & - & 7.2 & 4.8 \\
\hline
\end{tabular}

${ }^{a}$ Wall units were not specified in the references, so numbers given in the table are estimated by the authors and represent the likely maxima.

number which was set to 0.9 (Walton and Cheng, 2002; Xie et al., 2004). $k_{m}$ is the molecular diffusivity and is given by $v / S c_{m}$, where $v$ is the kinematic viscosity of the fluid. A molecular Schmidt number $\left(S c_{m}\right)$ of 0.71 was used; this is similar to that used in previous DNS studies of similar cases (e.g. Brethouwer et al., 1999; Vrieling and Nieuwstadt, 2003; Fabregat et al., 2009). $S$ is the scalar source of volume flux that was kept constant in time. The line source was aligned in the spanwise direction as shown in Fig. 1 which summarises the geometry. Cyclic boundary conditions were employed in the streamwise and in the lateral directions, but a zero scalar flux was imposed over a $y-z$ plane near the inlet to re-impose zero concentration upstream of the source. On the channel walls, the normal gradient of the scalar was set to zero, ensuring a zero surface flux.

A finite volume approach was used to discretize the flow and scalar equations, with a second-order central difference scheme for spatial discretization of Eq. 1. In order to eliminate any possible numerical instabilities, the monotone advection and reconstruction scheme (STAR-CD, 2009) with second-order accuracy and a blending factor of 0.99 was used for Eq. 2. A second-order backward implicit scheme was used for time discretisation.

Table 1 lists the computational domain sizes and the resolution at typical regions for $R e_{\tau}$ $=180$ and 520, with $\Delta x_{i}^{+}=\Delta x_{i} u_{\tau} / v$, where $\Delta x_{i}$ is the grid spacing in the $i$-th direction and $u_{\tau}$ is the friction velocity. In the streamwise and spanwise directions, the grid was uniform, whilst in the transverse $(y)$ direction the grid was stretched with finer resolution in the near wall region. The wall units at the channel centre and in the near wall region are denoted by $\Delta y_{c}^{+}$and $\Delta y_{w}^{+}$respectively. The computational domain consisted of hexahedral cells and the 
Table 2 Details of the source size and shape for $R e_{\tau}=180$

\begin{tabular}{lccc}
\hline Computations type & \multirow{2}{*}{ Source shape } & $2.4 \sigma_{s} / h\left(2.4 \sigma_{s}^{+}\right)$ & $H_{s} / h\left(H_{s}^{+}\right)$ \\
\hline LES & Gaussian & $0.0768(13.8)$ & - \\
DNS (Brethouwer and Nieuwstadt, 2001) & Rectangular & - & $0.1101(20.0)$ \\
DNS (Fabregat et al., 2009) & Rectangular & - & $0.028(5.0)$ \\
DNS (Vrieling and Nieuwstadt, 2003) & Rectangular & - & $0.054(9.7)$ \\
\hline
\end{tabular}

$\sigma_{s}$ is the standard deviation of Gaussian distribution of the 'Gaussian' source. $H_{s}$ is the size of the 'Rectangular' source in which a constant distribution is specified. In terms of wall units, $2.4 \sigma_{s}^{+}=2.4 \sigma_{s} u_{\tau} / v$ and $H_{s}^{+}=H_{s} u_{\tau} / v$. Dimensionless Kolmogorov length $\eta^{+} \equiv \eta u_{\tau} / v \sim 2$ (Kim et al., 1987)

flow was maintained by a constant pressure difference $\Delta p$ given by

$$
\Delta p L_{y}=2 \tau_{w} L_{x}
$$

where $L_{x}$ and $L_{y}$ are the domain lengths in the streamwise and transverse directions respectively and $\tau_{w}=\rho u_{\tau}^{2}$ is the wall shear stress. The time-step was small enough that the mean Courant number $(\Delta t U / \Delta x)$ was less than one. All the computations were carried out using STAR-CD (2009) version 4.08 (from CD-adapco). Some of the computations were run on Iridis, a local supercomputer at the University of Southampton and some on HECToR, one of the machines at the UK's supercomputer centre. For all the computations, the initial durations were more than $50 h / u_{\tau}$. The averaging durations for the flow and scalar were respectively $50 h / u_{\tau}$ and $20 h / u_{\tau}$. All the statistics were obtained by averaging in the spanwise direction as well as in time.

\section{Line source in a channel flow at $\operatorname{Re}_{\tau}=180$}

Before proceeding with the simulations at $R e_{\tau}=520$, a verification study was conducted in which the passive scalar dispersion from a line source placed at the centre of the channel at $R e_{\tau}=180$ was simulated using LES. The mean flow and fluctuations are compared with two DNS studies (Moser et al., 1999; Fabregat et al., 2009) in Fig. 2. The differences in the mean flow and fluctuations between the two DNS studies and the current LES may be attributed to the different numerical schemes and grid resolution, identified in Table 1. Note in particular that the grid spacings in the LES are finer in the streamwise direction and in the wall-normal direction at the channel centre compared with those in the two DNS studies, so the present LES (at this $R e_{\tau}$ ) could be viewed as almost a DNS. Nonetheless, the discrepancy in $\overline{v^{\prime 2}} / u_{\tau}^{2}$ between the LES and the DNS is probably due to the unresolved subgrid scale motions near the wall in LES that are not included in the figure and numerical scheme. Overall however, the LES velocity field is in reasonably good agreement with that given by DNS.

To study the shape effects of the line source, two sets of computations were performed. In the first set a constant $S$ in Eq. 2 was specified over the source cells; this is called the 'rectangular' source. In the second set, $S$ was specified via a Gaussian distribution in the $y$ direction over the source cells and this is denoted as the 'Gaussian' source. Both sources were placed at the channel centre as shown in Fig. 1. Details of the source size and shape used in LES and in the previous DNS studies are given in Table 2.

We compare the mean scalar field from LES and DNS. The width of the mean scalar $\bar{c}(y)$ plume is quantified by the profile's standard deviation $\left(\sigma_{c}\right)$ and its variation with streamwise 

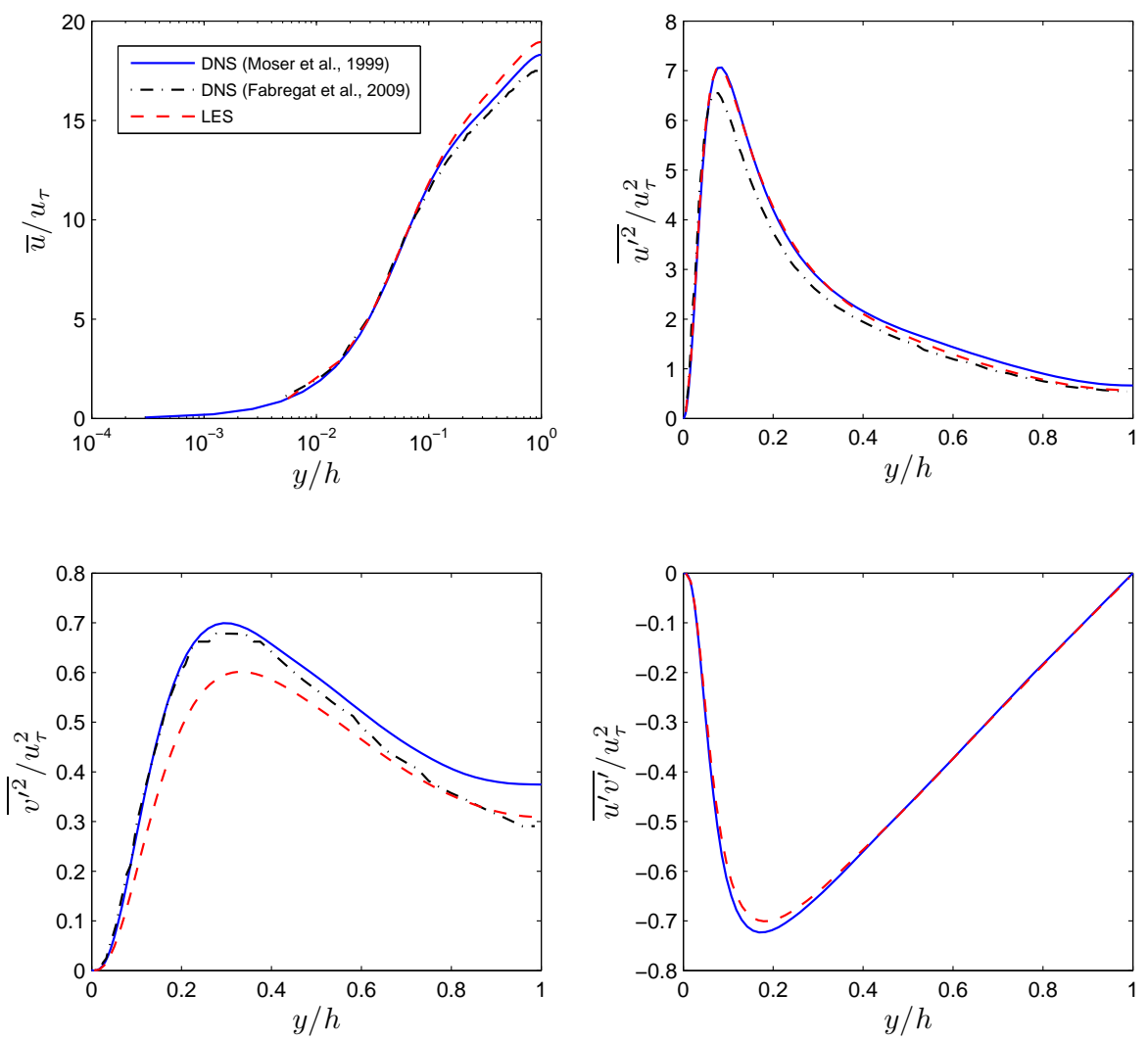

Fig. 2 LES streamwise mean velocity and Reynolds stresses at $R e_{\tau}=180$, compared with DNS data.

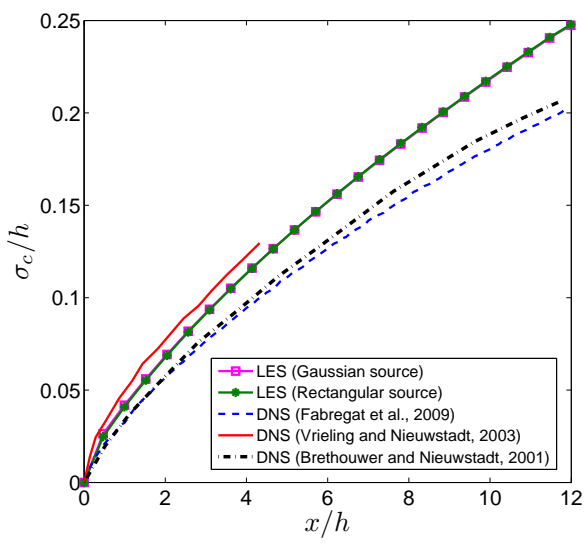

Fig. 3 Variation of the standard deviation of the plume with downstream distance.

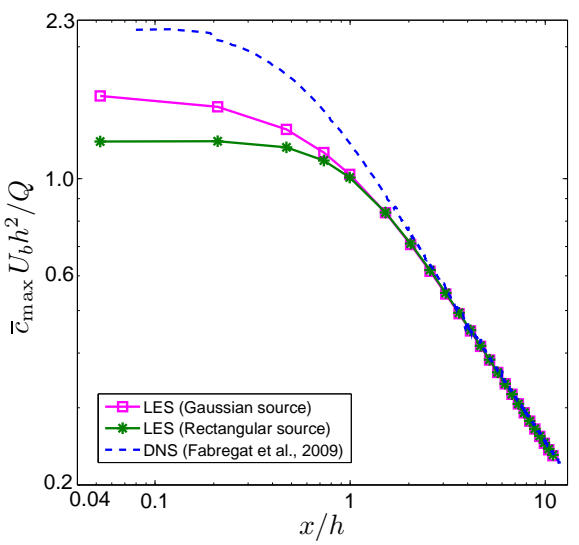

Fig. 4 Variation of maximum concentration (normalised with the scalar total flux $Q$ and the bulk velocity $U_{b}$ ) with downstream distance. 
distance is shown in Fig. 3. The standard deviation is corrected using its value at the source, i.e.

$$
\sigma_{c}=\sqrt{\sigma^{2}-\sigma_{0}^{2}}
$$

where $\sigma_{0}$ is the standard deviation of the source. The variance $\sigma^{2}$ is obtained using

$$
\sigma^{2}=\frac{\int_{0}^{2 h}(y-\beta)^{2} \bar{c}(y) \mathrm{d} y}{\int_{0}^{2 h} \bar{c}(y) \mathrm{d} y}, \quad \text { where } \quad \beta=\frac{\int_{0}^{2 h} y \bar{c}(y) \mathrm{d} y}{\int_{0}^{2 h} \bar{c}(y) \mathrm{d} y} .
$$

This method is simple and easy to use. Note that for the near wall source cases examined in $\S 4$, the standard deviation $\sigma$ calculated from the above equations may differ somewhat from that obtained by considering the profile as a combination of a Gaussian profile with its wall reflection from an imaginary source (Fackrell and Robins, 1982). Normally the former is less than the latter. It is observed in Fig. 3 that the values of $\sigma_{c}$ differ between the three DNS studies. The reason for this is unclear, but since the source size is taken into account by correcting with its value at the source, we must assume that the discrepancies in $\sigma_{c}$ arise solely because of the specifics of grid resolutions and numerical schemes, which may have led to slightly different mean flow and turbulence statistics (which were not shown by two of the three authors).

Figure 4 shows the downstream decay of the maximum mean concentration normalised with the scalar total flux $Q$. As usual, the half-width of the Gaussian profile is defined to be half the width of the profile where the mean concentration is half its maximum value; this is about 1.2 times the standard deviation. The source size for the Gaussian source is defined to be $2.4 \sigma_{s}(\sim 7 \eta$, where $\eta$ is the Kolmogorov length) whilst for the rectangular source it is $H_{s}(\sim 10 \eta)$. The effects of the source size and shape are evident only up to $x / h \leq 2$. Good agreement is observed between the LES and the DNS downstream of $x / h=2$. We conclude that the effect of source size (at least if less than $10 \eta$ ) and shape on the mean concentration is negligible in the far field for relatively low $R e$ number flows. Overall, this $R e_{\tau}=180$ simulation provides satisfactory verification of the LES code used.

\section{Line sources in a channel flow at $R e_{\tau}=520$}

Lavertu and Mydlarski (2005), hereafter denoted by LM, conducted experiments on the flow in a high-aspect-ratio channel whose dimensions were $267 h \times 2 h \times 37 h$ where $h=0.03 \mathrm{~m}$ is the half channel height. They studied passive scalar dispersion from three line sources in this channel. Each source comprised an electrically heated fine Nichrome wire stretched across the channel in the spanwise direction (which was vertical in their rig), but with temperatures sufficiently small to ensure that the source was essentially passive. Source locations were $y_{s} / h=0.067,0.17$ and 1 , for which $y_{s}^{+}=y_{s} u_{\tau} / v$ were 35,87 and 520, respectively. The present computations used the same source locations, but the code allowed Eq. 2 to be solved for three separately identifiable scalars simultaneously; this avoided having to undertake the entire computation three times. Before considering the behaviour of the scalar plumes, we present details of the mean velocity and turbulence fields. 
Table 3 Values of the source size, taken as $2.4 \sigma_{s} / h$, at the three source locations. Values in brackets refer to $2.4 \sigma_{s}$ in wall units $-2.4 \sigma_{s} u_{\tau} / v$ for $R e_{\tau}=520$, except for the entry labelled 'a', for which the source was rectangular (size $H_{s}^{+}$) and covered one grid cell.

\begin{tabular}{lccc}
\hline & $y_{s} / h=0.067$ & $y_{s} / h=0.17$ & $y_{s} / h=1$ \\
\hline Set 1 (small line source) & $0.0156(8.2)$ & $0.012(6.2)$ & $0.032(16.6)^{\mathrm{a}}$ \\
Set 2 (large line source) & $0.024(12.5)$ & $0.024(12.5)$ & $0.022(11.5)$ \\
\hline
\end{tabular}

\subsection{Flow field}

Details of the computational domain and the resolution used in this study and in the earlier DNS study (Moser et al., 1999, for the flow only) are given in Table 1. The streamwise mean velocity $\bar{u}$ and the Reynolds normal and shear stresses in the $x-y$ plane obtained from LES and normalised using $u_{\tau}$ are compared with the DNS and the experiments in Fig. 5. The LES $\bar{u}$ profile lies between the DNS and the experimental data with the maximum difference at the centre of the channel. In contrast to this, LES velocity profile lies above DNS at $R e_{\tau}=180$ in Fig. 2. Comparing these two flow fields, it can be observed that increase in the values of $\operatorname{Re}_{\tau}$ led to increase in the velocities (as $h$ and $v$ are constant). In Fig. 5, the flow field from DNS corresponds to $R e_{\tau}=590$ and that of LES at $R e_{\tau}=520$. This could be the reason for slightly smaller values of $\bar{u}$ in LES compared with DNS. Near the wall the resolved streamwise fluctuations are slightly over-predicted whereas at the centre-line they are marginally underpredicted by LES compared with DNS. In the experiments, the error in obtaining $u_{\tau}$ may be as much as $\pm 4 \%$ (L. Mydlarski, pers. comm, 2010) which could account for some of the discrepancies with the DNS data. The slightly smaller discrepancies between the LES and experiments may be reflected in the scalar dispersion data, as discussed later. Subgrid stresses probably account for the rather lower values of $\overline{v^{\prime 2}}$ predicted by the LES compared with the DNS. Differences between the LES and DNS shear stress profiles are very small and, overall, the flow field comparisons between the LES and both experiments and DNS are sufficiently good to give confidence in the scalar predictions discussed next.

\subsection{Scalar field}

In the experiments, the Nichrome wires had diameters $d_{s}=1.27 \times 10^{-4} \mathrm{~m}$ for measurements close to the source $(x / h \leq 10.8)$ and $2.54 \times 10^{-4} \mathrm{~m}$ for measurements farther downstream, to improve the signal-to-noise ratio. The former diameter corresponds to $4.2 \times 10^{-3} \mathrm{~h}$, or about $0.45 \eta$ at $R e=10400$, where $\eta$ is the Kolmogorov length scale at the channel centreline. (For the near-wall source location, the corresponding value was about $0.85 \eta$.) LM concluded that their results were independent of the source diameter in that range for the downstream locations under consideration. In the present LES, the length of the streamwise domain was $4 \pi h$ and hence the scalar field with the experiments could be compared up to $x / h \approx 11$. The sources were located around $0.3 h$ downstream of the inlet plane. It would have been computationally too expensive to model the exact geometry of such a small line source, so two different sets of computations were done with different source sizes in order to study the source size effect on the scalar dispersion before comparing with the experiments. The details of both sets are given in Table $3^{1}$. The Gaussian source sizes (i.e. $2.4 \sigma_{s}$ ) for Set 1 and Set 2 are about $3 \eta$ and $5 \eta$ respectively, assuming $\eta^{+}=2.7(\mathrm{LM})$.

\footnotetext{
1 Note that the size of the sources at $y_{S} / h=1$ is not reflected solely in the values shown due to their different shapes.
} 

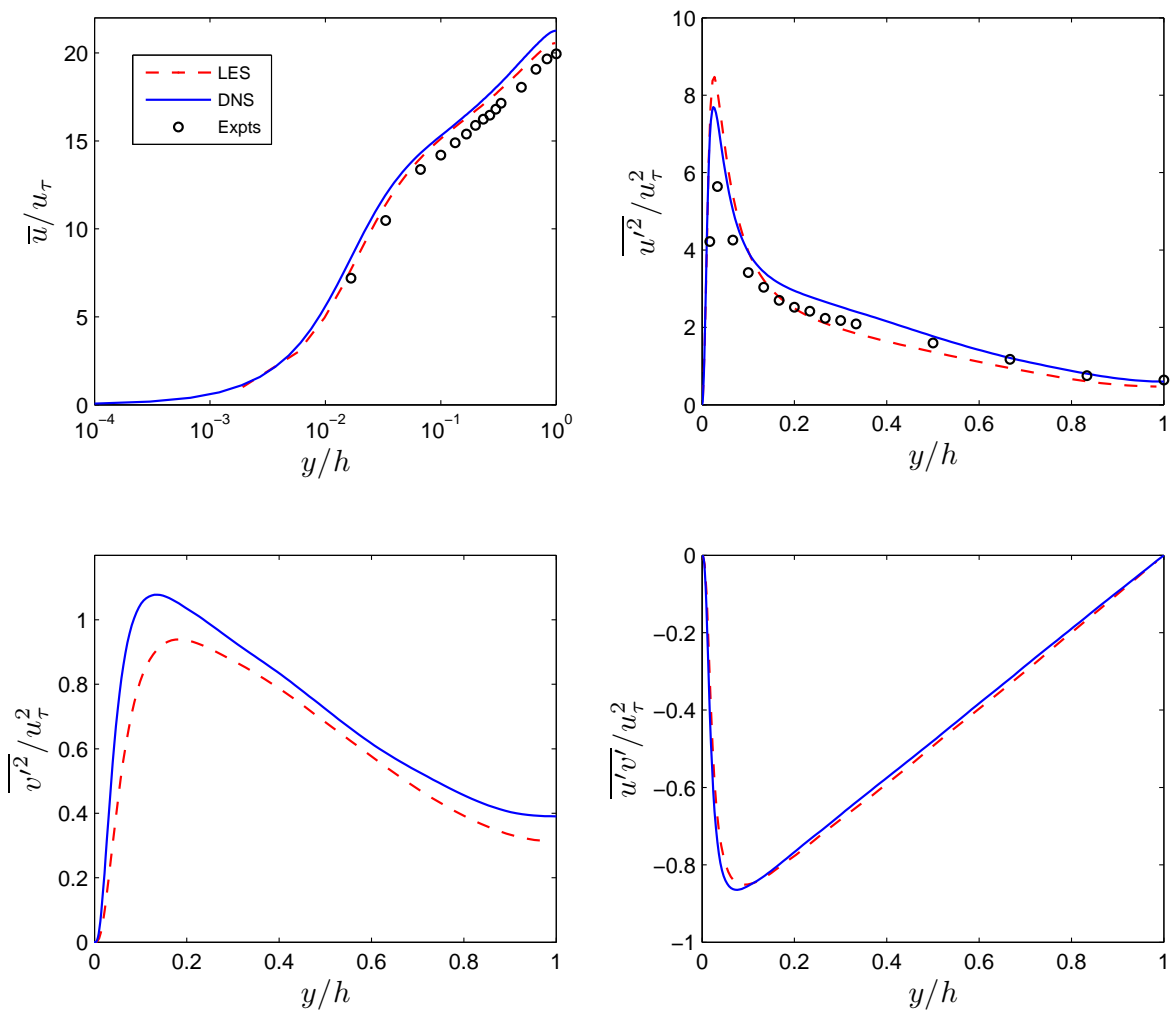

Fig. 5 Comparison of streamwise mean velocity and the Reynolds stresses with DNS (Moser et al., 1999) and experiments (Lavertu and Mydlarski, 2005) for $R e_{\tau}=520$.

Instantaneous profiles of the scalar plume for the three line sources from Set 2 are shown in Fig. 6. The bulk flapping of the scalar wake is evident immediately downstream of all three line sources. Even for the centre-line source, it can be observed in Fig. 6a that the scalar has not yet reached the side walls far downstream and plume meandering is observed throughout the domain length. For the two near-wall sources Fig. 6 shows that, not surprisingly, the plumes reach the bottom wall relatively quickly and earliest for the source nearest to the wall.

The time series of the normalised scalar from the three line sources at their respective source heights and at $x_{c} / h=7.4$ are shown in Fig. 7. Two dotted lines are included at $c / \bar{c}=$ 0.5 and 1.5 in order to show clearly the frequency of the large excursions in concentrations from its mean value. As expected, most of the scalar lies within this range for the near wall sources and not for centre line source due to the flapping nature of the latter plume. This shows that the intermittent nature of the plumes from all these sources are different; details are discussed in $\S 4.2 .3$. 


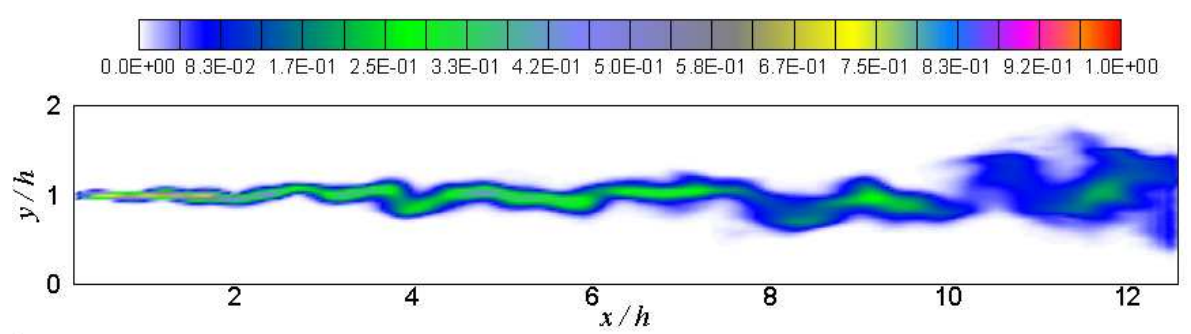

(a) $y_{s} / h=1$

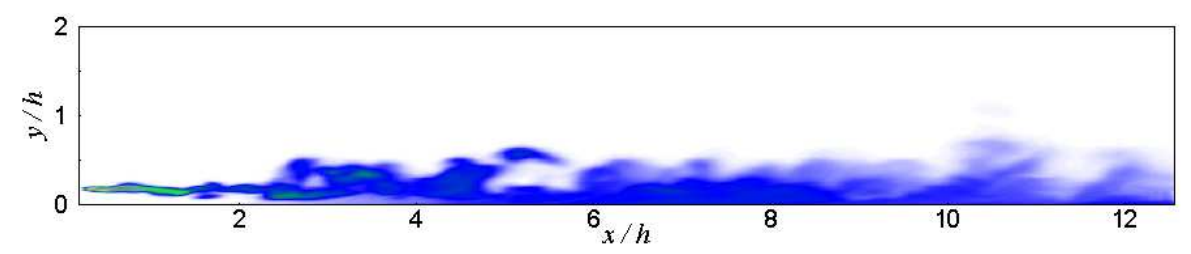

(b) $y_{s} / h=0.17$

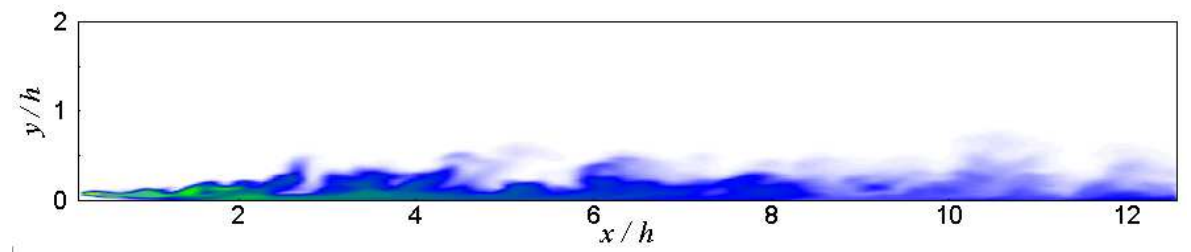

(c) $y_{s} / h=0.067$

Fig. 6 The instantaneous contours of the scalar normalised by the mean concentration at the source centre for the three line sources from Set 2.

\subsubsection{Mean concentration of scalar}

For the range of source size considered in Table 3, we noticed that the calculated mean concentration is independent of the size after certain downstream distance which, given the results of Fackrell and Robins (1982) and Xie et al. (2004), is perhaps not surprising. However in the region nearer to the source, the mean concentration is clearly very dependent on source size and shape. A virtual origin correction is made for the two sets of line sources by fitting linear curves to the growth in standard deviation $(\sigma / h)$ of the mean concentration profile, as shown in Fig. 8. According to Anand and Pope (1985) the development of the mean scalar field in high $R e$, non-decaying, homogeneous turbulence consists of the following three stages:

$$
\begin{array}{ll}
\text { Molecular diffusion, } t \ll \Gamma \overline{{v^{\prime 2}}^{2}}: & \sigma^{2} \approx 2 \Gamma t \\
\text { Turbulent convection, } \Gamma / \overline{v^{\prime 2}} \ll t \ll T: \sigma^{2} \approx \overline{v^{\prime 2}} t^{2} \\
\text { Turbulent diffusion, } t \ll T: & \sigma^{2} \approx 2 \Gamma_{t} t
\end{array}
$$

Here, $t$ is the diffusion time, $\Gamma$ is the thermal or molecular diffusivity, $v^{\prime}$ is the Lagrangian velocity fluctuation, $T$ is the Lagrangian integral time scale and $\Gamma_{t}$ is the turbulent diffusion coefficient. In our computations, the molecular diffusion stage near the source is very short and this probably is a result of the relatively large source size. The turbulent convection stage prevailed in the computational domain to some extent where the plume growth is linearly 
(a) $y_{s} / h=0.067$

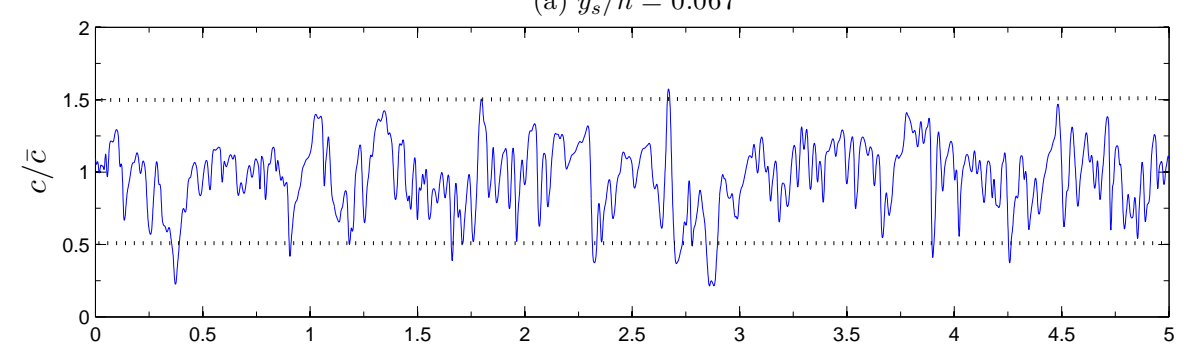

(b) $y_{s} / h=0.17$

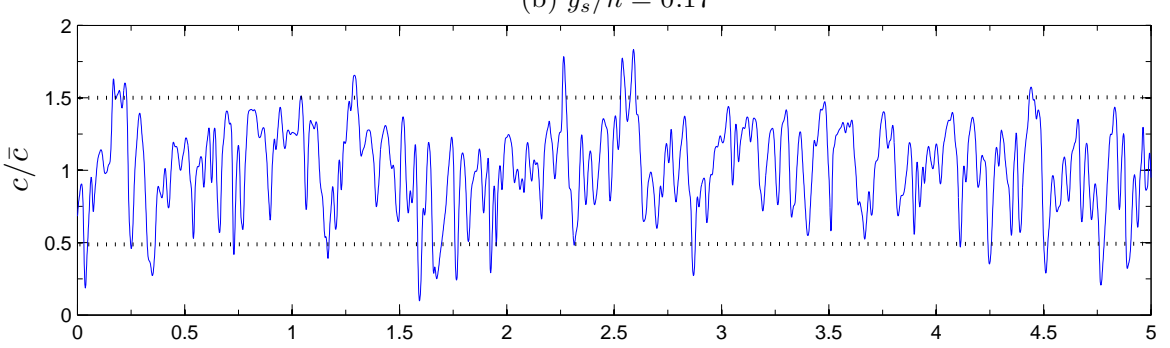

(c) $y_{s} / h=1$

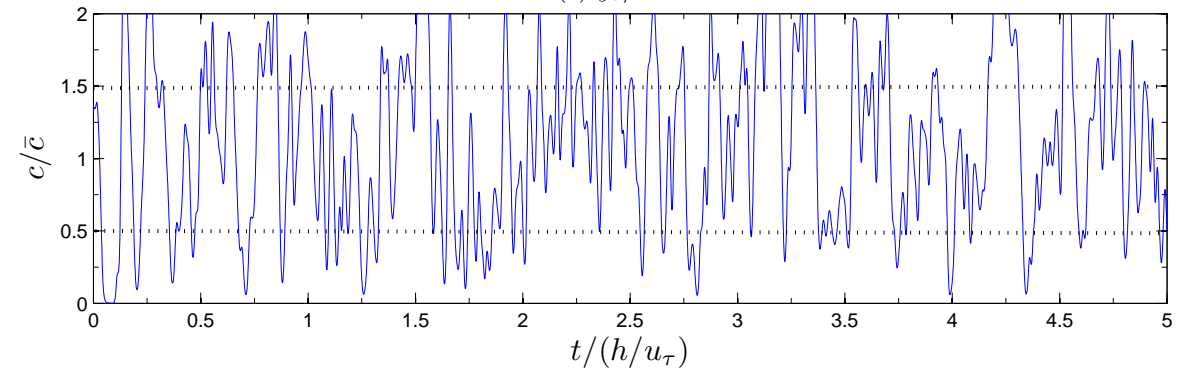

Fig. 7 Time series of the scalar from three line sources at their respective source heights and at $x_{c} / h=7.4$.

proportional to the diffusion time (see Fig. 8). The virtual origins of the line sources, $x_{o} / h$, correspond to the stations where $\sigma / h=0$.

Figure 9 compares the maximum mean concentration $\bar{c}_{\max }$ normalised with the respective source total flux $Q$ for the two sets of line sources at the three different wall normal locations. The abscissa corresponds to the streamwise distance corrected for the virtual origin i.e. $x_{c} / h=\left(x-x_{o}\right) / h$. The decays from the two sets of data are in good agreement for the two near wall sources. Notice the small 'bumps' in these profiles; these correspond to the locations where $\bar{c}_{\max }$ reaches the bottom wall, which is at $x_{c} / h \approx 6$ and 2 for $y_{s} / h=0.17$ and 0.067 respectively. In Fig. $9 \mathrm{c}$, the profiles of $\bar{c}_{\max }$ for the two sources do not collapse in the near source region, e.g. for $x_{c} / h \leq 2$, which is anticipated because the shape effects of the source cannot be mitigated merely by using a virtual origin correction. (Recall that for the centre-line location, the smaller source has a rectangular shape and the larger one has a Gaussian shape.) In order to compare the LES mean scalar quantities with the experimental 


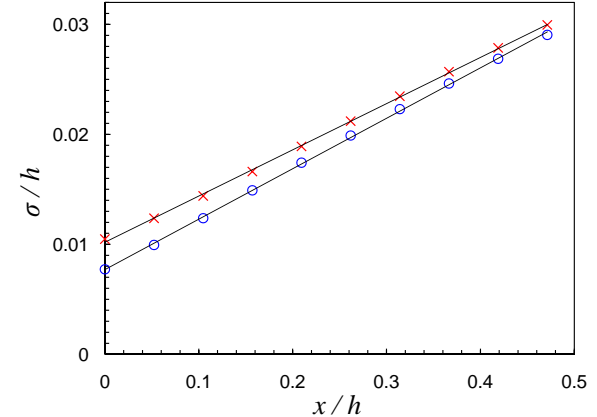

(a) $y_{s} / h=0.067$

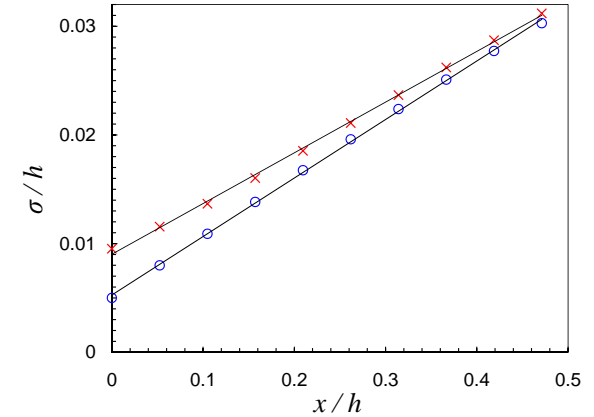

(b) $y_{s} / h=0.17$

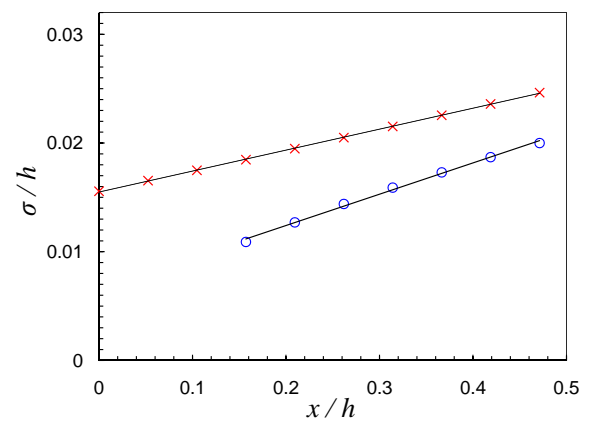

(c) $y_{s} / h=1$

Fig. 8 Determining the virtual origin $\left(x_{0} / h\right)$ for the three line source locations. $\times$ : large source; $\circ$ : small source.

results, the data from either of these two sets can be considered, with an appropriate correction for the source size. We use the data from Set 2 (the larger source) for the comparisons.

Figure 10 shows the normalised profiles of the mean concentration for the three line sources. It is observed in all cases that the scalar profiles from the experiments are slightly wider than those from LES. Bakosi et al. (2007), however, found that the IECM overpredicted the plume width at the far downstream stations for the sources at $y_{s} / h=0.067$ and 1 and at $R e_{\tau}=1080$. They stated that due to an under-prediction of the wall normal Reynolds stress component $\overline{v^{\prime 2}}$ by the velocity model and the uncertainties in the experimental data, the mean scalar profiles should be considered only qualitatively. But it is not quite clear how the under resolved transverse fluctuations could over-predict and underpredict the plume width in PDF model and LES respectively. The disagreement of plume widths in LES and experiments could be due to any or combination of the following: underresolved transverse fluctuations in LES, over-prediction of maximum mean concentration in LES due to advection, and uncertainty in the measurement of reference mean temperature in the experiments (LM).

In Fig. 10a, it is observed that the peak of the mean concentration from LES is at the wall for $x_{c} / h \geq 4$, whereas the data from experiments have peaks initially at the source height (at $\left.x_{c} / h=4\right)$ and then gradually nearer the wall for distances farther downstream. In Fig. 10b, at $x_{c} / h=4$, LES shows the peak of the plume at $y / h=0.12$ which is lower than 


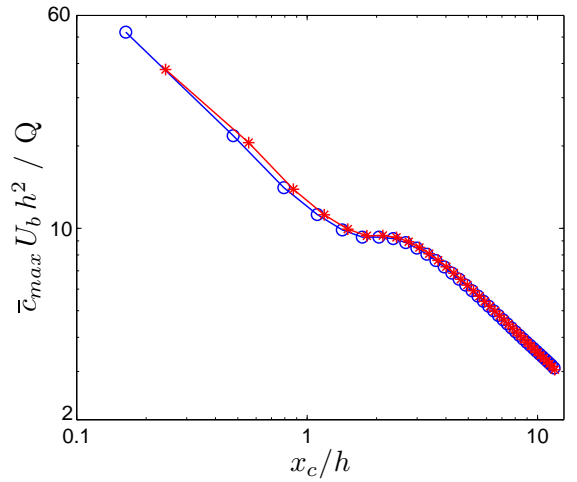

(a) $y_{s} / h=0.067$

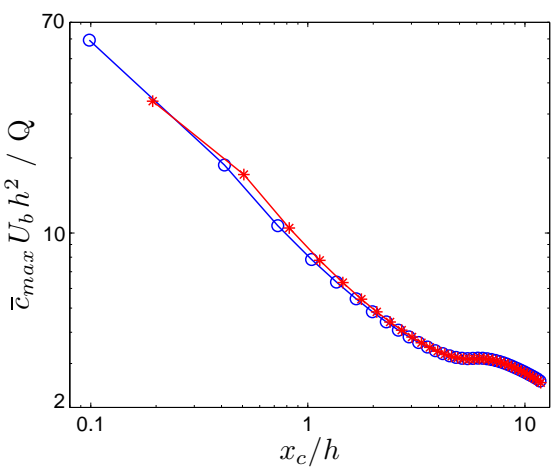

(b) $y_{s} / h=0.17$

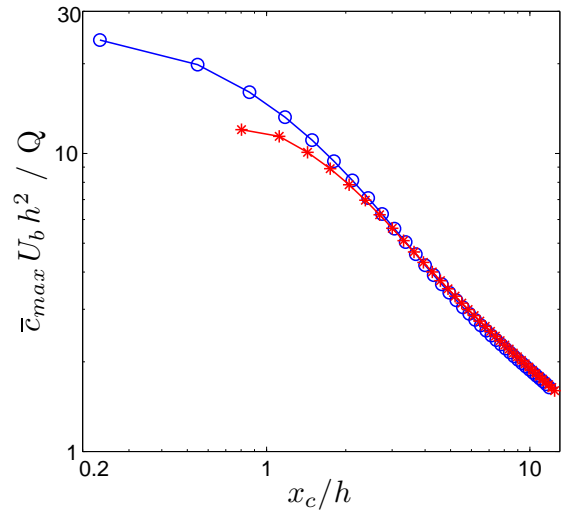

(c) $y_{s} / h=1$

Fig. 9 The decay of the maximum mean concentration (normalised with the scalar total flux $Q$ and the bulk velocity) along the downstream distance. $*$ : large source; $\circ$ : small source.

the source height. This is consistent with the previous experimental and LES data (Fackrell and Robins, 1982; Sykes and Henn, 1992) for a point source at $z_{s} / H=0.19$ in a turbulent boundary layer flow with boundary layer thickness $H$. Also, in experiments for a line source in uniform shear turbulent flow (Karnik and Tavoularis, 1989), the peak of the mean scalar was found to be shifting towards the lower velocity regions at far downstream distances. For adiabatic wall conditions, in principle the profile of mean concentration should be given by superposition of a Gaussian profile and its reflection in the wall (i.e. an imaginary source at $y=-y_{s}$ ). The peak of the mean concentration profile should then be at the wall provided (roughly) that $\sigma_{c} \geq y_{s}$. For the near wall sources $y_{s} / h=0.067$ and 0.17 , these locations correspond to $x / h \approx 2$ and 6 , respectively, so the profile peak locations from the LES data are consistent with this expectation, as confirmed in Figs. 6, 10a, 10b and 11. In contrast, the experimental peak locations for the source $y_{s} / h=0.17$ are not at $y=0$, as noted above, even far downstream. It is speculated that this must be due either to the lack of near-wall data, wall-probe interference effects, experimental scatter, or the lack of a wall temperature measurement, or a combination of these (L. Mydlarski, priv. comm, 2010). 

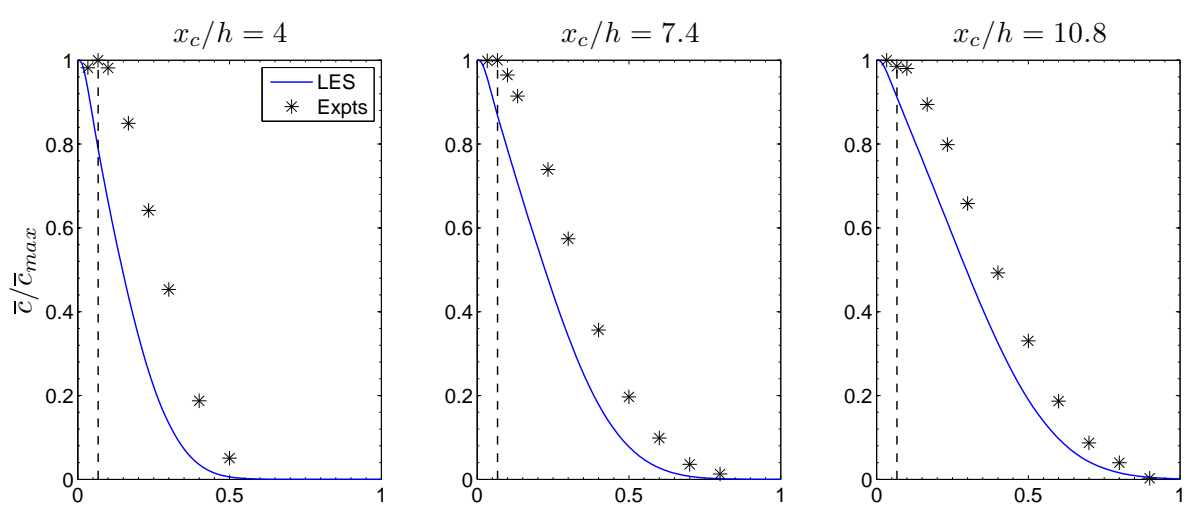

(a) $y_{s} / h=0.067$
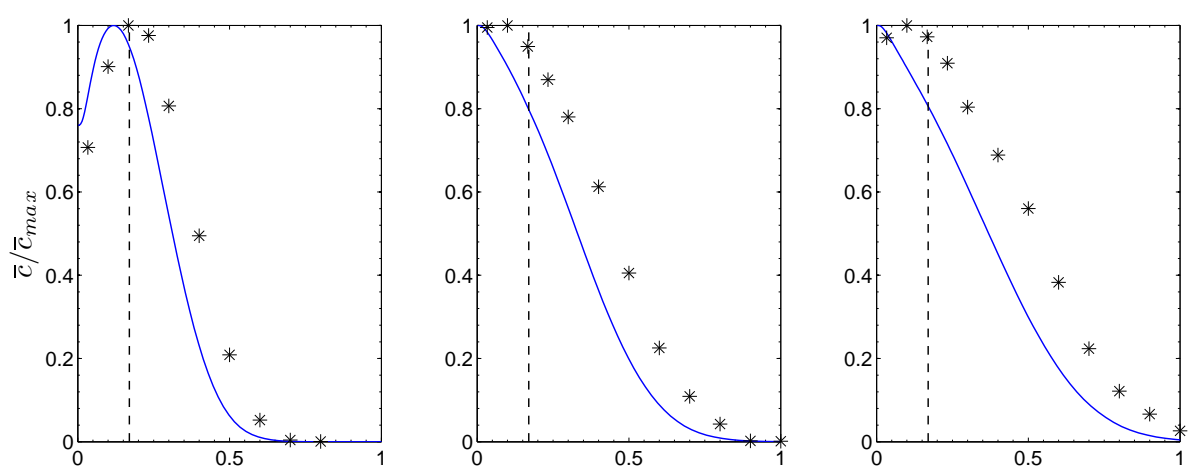

(b) $y_{s} / h=0.17$
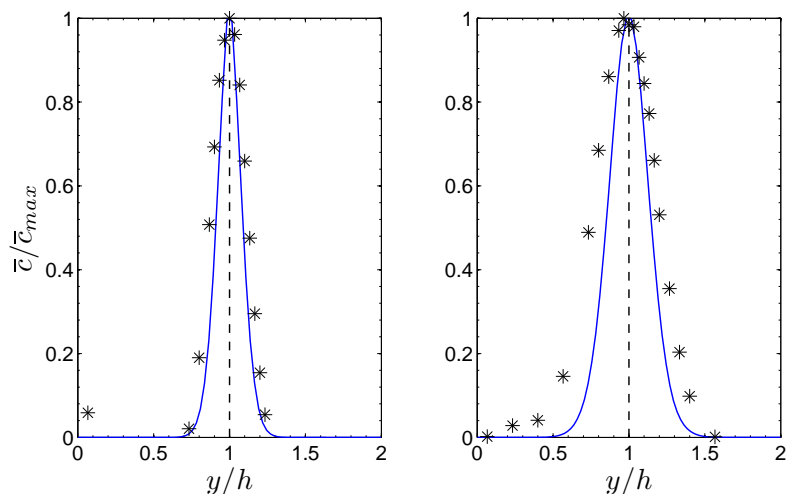

(c) $y_{s} / h=1$

Fig. 10 Normalised mean concentration profiles at typical downstream locations. The dashed lines represent the transverse locations of the line source. 


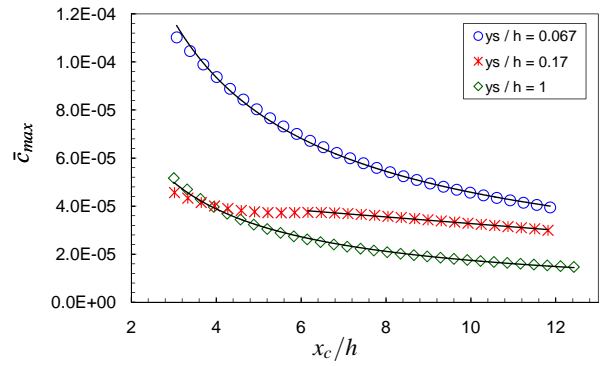

Fig. 11 The decay of the maximum mean concentration with the downstream distance. Solid lines are power law fits with exponents given in Table 4.

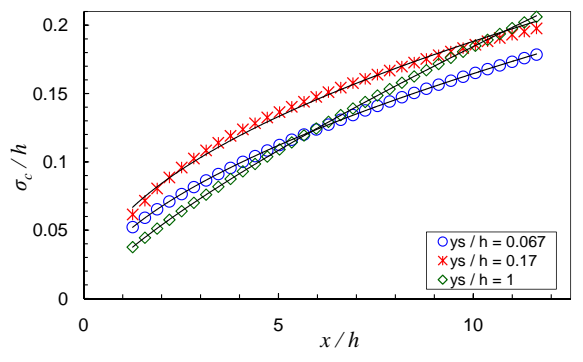

Fig. 12 The growth of the mean plume width with the downstream distance. Solid lines are power law fits with exponents given in Table 4.

Table 4 The exponent of the power law fit to the curves of the (i) maximum mean concentration profiles: $\bar{c}_{\text {max }} \propto\left(x_{c} / h\right)^{n}$ and (ii) the standard deviation of the mean concentration profiles: $\sigma_{c} / h \propto(x / h)^{n}$

\begin{tabular}{|c|c|c|c|c|}
\hline & \multicolumn{3}{|c|}{$n$} \\
\hline & & $y_{s} / h=0.067$ & $y_{s} / h=0.17$ & $y_{s} / h=1$ \\
\hline \multirow{3}{*}{ (i) } & LES (Set 2) & -0.782 & - & -0.875 \\
\hline & Expts (LM) & -0.7 & -0.5 & -0.6 \\
\hline & Expts (Karnik and Tavoularis, 1989) & - & - & -0.75 to -1.0 \\
\hline (ii) & LES (Set 2) & 0.5548 & 0.4996 & 0.7664 \\
\hline
\end{tabular}

Figure 10c shows that for the centre-line source the plume profiles from the LES seem to be in fair agreement with the experiments at $x_{c} / h=4$ but not at $x_{c} / h=7.4$. It is stated in LM that there are inaccuracies in measuring the free-stream temperature, which gave rise to low signal-to-noise ratios in the measurement of the mean temperature excess profiles (particularly far downstream), so the authors emphasised the qualitative, rather than quantitative behaviour of their profiles. Recall also that the velocity fluctuations are slightly underestimated and the mean velocity slightly over-predicted by the LES when compared to the experiments (Fig. 5), both of which could lead to somewhat narrower plumes. All these factors could explain the differences between the simulations and the experiments.

The variation of the maximum mean concentration $\bar{c}_{\text {max }}$ with the streamwise distance for all the three lines sources (Set 2) are shown in Fig. 11. Note that the $\bar{c}_{\text {max }}$ are not normalised, in order to be consistent with the experiments. For the near wall and centre line sources, the data from $x_{c} / h=3$ could be fitted with a power law. The data for $x_{c} / h \geq 6$ can be fitted using a linear curve but no such fit is possible if the data are considered upstream of $x_{c} / h=6$ (since the peak has not yet reached the wall). Table 4 compares the power law exponent $n$ obtained from the simulations with those of the experiments by LM and Karnik and Tavoularis (1989). The LES data for the source at $y_{s} / h=0.17$ did not show any power law dependence.

From these comparisons in Table 4 an interesting observation can be made: the peak mean concentrations for sources near the wall and at the centre of the channel decay similarly to what is observed in a homogeneous uniform shear turbulent flow. The LES data suggests that the decay rate is faster for the centre line source compared with that for the near wall source. This could be due to the wall impeding plume development in the latter case, resulting in less meandering than occurs for the centre-line plume. The fact that decay of the peak mean concentration from the source at $y_{s} / h=0.17$ follows a different pattern 
than that for sources at $y_{s} / h=0.067$ and 1.0 is no doubt due to the interactions between the plume and the wall in the intermediate stages.

The normalised standard deviation $\sigma_{c} / h$ of the mean plume is calculated using Eqs. 3 and 4 and is shown in Fig. 12 for the three source locations. The power law is found to be a good fit from $x / h>1$ for all three cases. The width of the mean scalar profile in the experiments did not show any similar dependence with $x / h$. The power law exponent for the LES at $y_{s} / h=0.067$ and 0.17 data are both found to be approximately 0.5 and for the data from the centre line source it is found to be approximately 0.75 . It is observed in Fig. 12 that the mean plume width for the source at $y_{s} / h=0.17$ is greater than that for the near wall source as the plume growth of the latter is impeded by the bottom wall much earlier. The plume width for the centre line source is less than those for the two near wall sources up to $x / h<6$ but then gradually exceeds them. Note that the normalised standard deviation $\sigma_{c} / h$ develops from zero at $x / h=0$. Due to the low local turbulence intensity in the channel centre, slower plume growth is anticipated in the near source region. With increasing downstream distance, the meandering of the plume gradually becomes more significant and, unlike the near wall source cases, the plume is not impeded by the channel walls within the computational domain. Nonetheless, the edges of the plume gradually reach regions with significantly higher local turbulence intensity than on the centre-line.

\subsubsection{Concentration fluctuation}

The vertical profiles of the scalar fluctuations normalised with their maxima are compared with the experimental data in Fig. 13 at typical downstream distances for the three line sources. For the near-wall sources, apart from the location of the maximum $c_{r m s}$, the agreement in the vertical profiles of $c_{r m s}$ between LES and the experiments are better than those for the corresponding mean profiles in Fig.10, probably as a result of the higher accuracy in the scalar fluctuation data (as noted by LM).

For both the LES and the experiments, the peak $c_{r m s}$ drifts away from the wall with increasing downstream distance. This is due to the rapid mixing in the near wall region and the high scalar intermittency at the edge of the plume. This drift in peak location is observed to be greater in the experiments than it is in the LES, which could be a consequence of the drift in the peak of mean concentration in the experiments, as seen in Figs. 10a and $10 \mathrm{~b}$. The peak drift is more distinctive for the near wall source at $y_{s} / h=0.067$ than for the source at $y_{s} / h=0.17$. The LES $c_{r m s}$ profiles from the centre-line source are in reasonable agreement with the experiments (Fig. 13c), although the LES slightly under-predicts the width of the $c_{r m s}$ profile at all locations. This might be attributed not only to the slight underprediction of the resolved velocity fluctuations at the core of the channel but also the underestimated mean velocity in the experiments (Fig. 5). In Bakosi et al. (2007), the IECMpredicted $\overline{c^{\prime 2}}$ profiles for the source at $y_{s} / h=1$ are in fair agreement with the measurements. In contrast, the IECM data for the source at $y_{s} / h=0.067$ show an evident discrepancy with the measurements.

In the LES, double peaks are observed up to $x_{c} / h<1.5$ for $y_{S} / h=0.067$ and up to $x_{c} / h<3$ for $y_{s} / h=0.17$. They are not observed far downstream and this could be because of the impedance of the plume growth by the bottom wall. For the centre line source, the bulk flapping of the plume that is primarily responsible for the occurrence of the double peak near the source is not present, presumably due to the source size. Whilst the LES profile at $x_{c} / h \approx 4$ is symmetric with a single peak at the channel centre, the downstream profiles start to deviate from this as can be seen in the profile at $x_{c} / h=7.4$. Eventually, e.g. from $x_{c} / h>8$, double peaks begin to form and one such profile is shown at $x_{c} / h=10.8$. 

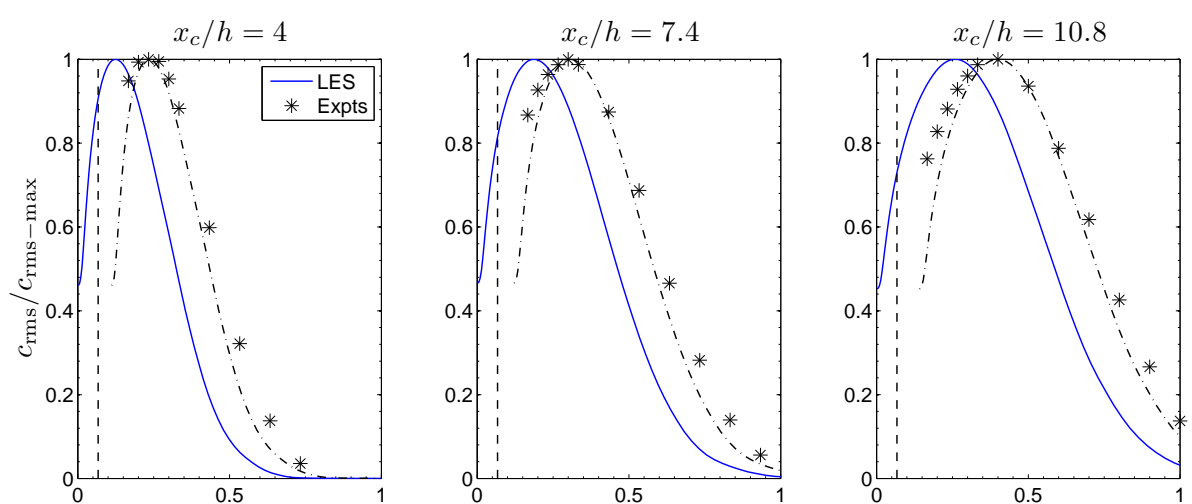

(a) $y_{s} / h=0.067$
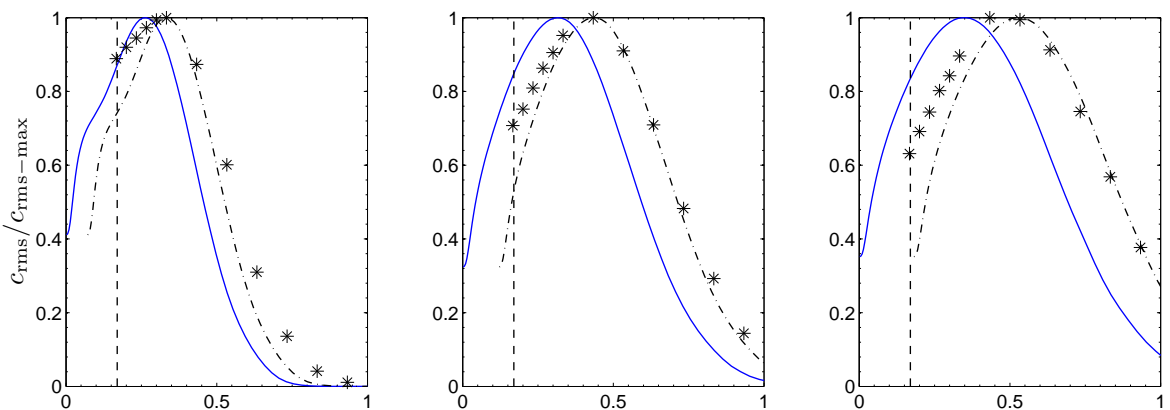

(b) $y_{s} / h=0.17$
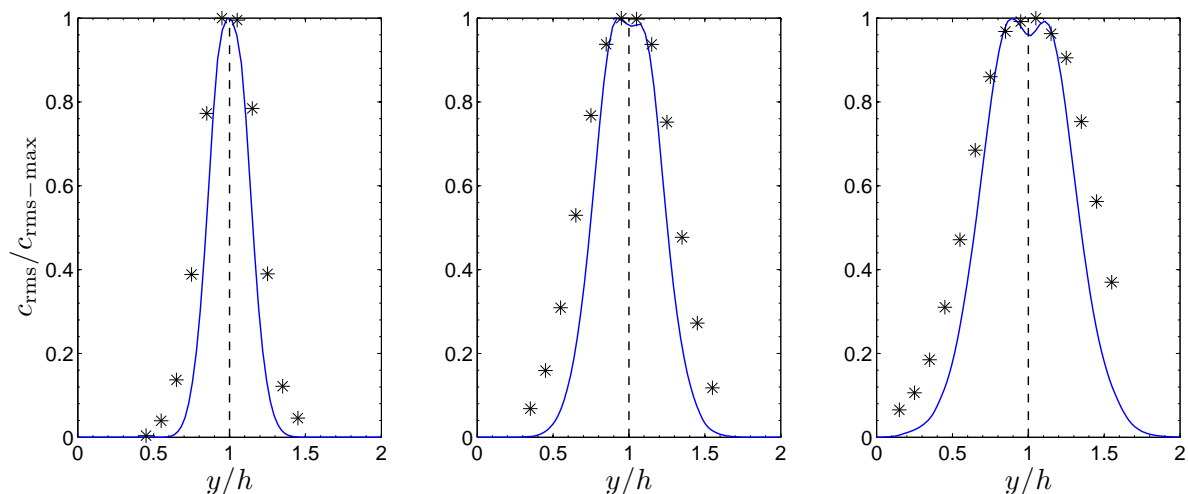

(c) $y_{s} / h=1$

Fig. 13 The r.m.s of the concentration fluctuation profiles normalised with their maxima at typical downstream locations. Dashed line represents the location of the line source. Dot-dashed profiles are the LES profiles with the peaks shifted to the experimental peak locations. 


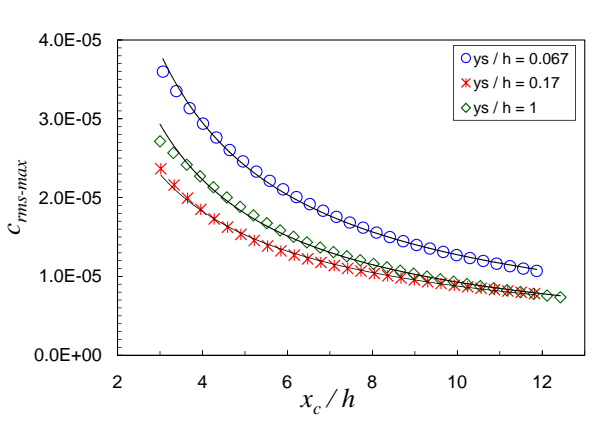

Fig. 14 The decay of the maximum r.m.s concentration fluctuation with the downstream distance. Solid lines are power law fits with exponents given in Table 5.

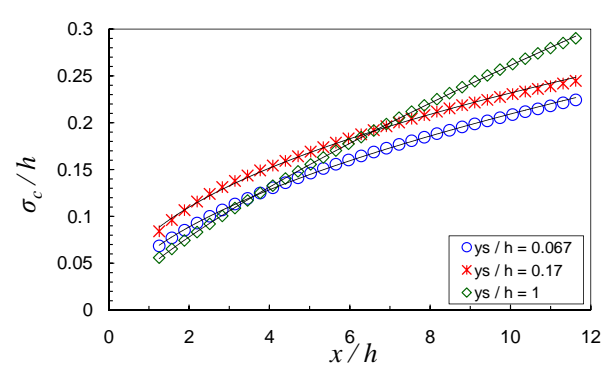

Fig. 15 The variation of the width of the r.m.s concentration fluctuation profiles with the downstream distance. Solid lines are power law fits with exponents given in Table 5.

Similar double peaks at far downstream locations from the line source are also observed for very large value of $S c_{s}$, thereby indicating that its effects are very small. It was observed in the grid turbulence experiments of Warhaft (1984) and in the uniform shear turbulence experiments of Karnik and Tavoularis (1989) that the emergence of a double peak in the $c_{r m s}$ profiles initially occurs very close to the source, but the peaks then merge to become a single peak in the intermediate region and a double peak again develops in the far field. The double peaks were also observed in the far field by Fabregat et al. (2009) in their DNS study but were not observed in the DNS study of Brethouwer and Nieuwstadt (2001). The absence of double peaks in the latter case could be a result of the relatively coarse resolution (see Table 1). In LM, the measurements were not made close enough to the source to observe double peaks in the $c_{r m s}$ profiles, but nor were such peaks observed far downstream. LM stated that this could have been because the plume widths were smaller than the integral scale of the turbulence, but if that were the cause we would not expect double peaks to appear in the LES data, since the mean flow and turbulence statistics are essentially the same as in the experiments. Also, the observed small ripple of double peak in LES is within the experimental error. Further understanding is required before these discrepancies between the simulations and the experiments can be fully explained.

The decay of the maximum concentration fluctuation $c_{r m s-\max }$ with downstream distance is shown in Fig. 14. A power law appears to be a good fit for the data from $x_{c} / h=3$ for all the three line sources. The exponents of the power law from the LES and the experiments (LM; Karnik and Tavoularis, 1989) are shown in Table 5. The standard deviations of the $c_{r m s}$ profiles are shown in Fig. 15 for the three line sources. A power law again seems to be a good fit for all the curves and the corresponding exponents are compared with the experiments (LM) in Table 5. The values of $n$ from LES are in fair agreement with the experiments. It is to be noted that the exponents of the mean and $c_{r m s}$ of the scalar are almost the same, which indicates that the plume growth rate of the scalar fluctuations is almost the same as that of its mean. A non-monotonic trend is observed in the values of $|n|$ with increase in $y_{s} / h$. It was suggested by LM that the decrease of $|n|$ for $y_{s} / h=0.17$ compared with that for $y_{s} / h=0.067$ is due to the decrease in turbulence intensity with increasing distance from the wall, whereas the increase of $|n|$ for $y_{s} / h=1$ is due to an increase in flapping of the plume. This certainly seems a reasonable explanation (recall the evidence of greater flapping for the centre-line source seen in Fig. 6). 
Table 5 The exponent of the power law fit to the curves of the (i) maximum r.m.s concentration fluctuation profiles: $\bar{c}_{r m s-\max } \propto\left(x_{c} / h\right)^{n}$ and (ii) the standard deviation of the r.m.s concentration profiles: $\sigma_{c} / h \propto(x / h)^{n}$

\begin{tabular}{llccc}
\hline & & & & \\
& & & & \\
& & $y_{s} / h=0.067$ & $y_{s} / h=0.17$ & $y_{s} / h=1$ \\
\hline \multirow{2}{*}{ (i) } & LES & -0.916 & -0.799 & -0.955 \\
& Expts (LM) & -1.311 & -0.952 & -1.133 \\
& Expts (Karnik and Tavoularis, 1989) ${ }^{\mathrm{a}}$ & - & - & $-0.85,-1.6$ \\
\hline (ii) & LES & 0.533 & 0.464 & 0.751 \\
& Expts (LM) & 0.431 & 0.372 & 0.679 \\
\hline
\end{tabular}

${ }^{\mathrm{a}}$ Near the source, the value was found to be -0.85 and -1.6 in the far field.

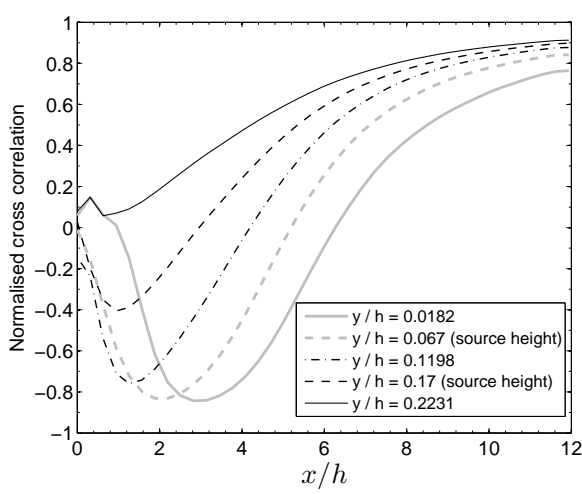

Fig. 16 Downstream variation of cross correlation of near wall scalars.

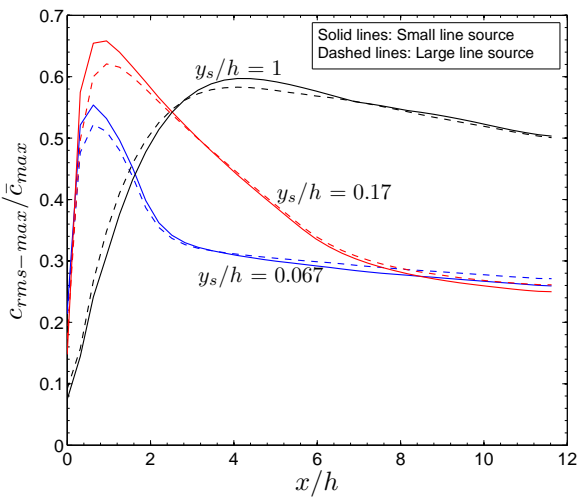

Fig. 17 Downstream development of relative intensity of concentration fluctuations.

The variation of the normalised cross correlation of the two near wall scalars, given by $\overline{c_{1}^{\prime} c_{2}^{\prime}} / \sqrt{\overline{c_{1}^{\prime 2}}} \sqrt{\overline{c_{2}^{\prime 2}}}$ with the downstream distance is shown in Fig. 16. Here $c_{1}^{\prime}$ and $c_{2}^{\prime}$ corresponds to the scalar fluctuation from the source at $y_{s} / h=0.067$ and 0.17 respectively. A detailed investigation on the correlation coefficient of thermal plume arising from a pair of line sources in a turbulent channel flow at $R e_{\tau}=510$ was made by Costa-Patry and Mydlarski (2008). In their experiments, various combinations of the wall normal positions of the line sources (represented by the average line source position from the wall, $y_{s_{a v}} / h$ ) and the separation distances of the line sources $(d)$ were considered and the correlation coefficients of relevant cases were compared with the DNS study of Vrieling and Nieuwstadt (2003) in turbulent channel flow, experiments of Warhaft (1984) in grid turbulence and experiments of Tong and Warhaft (1995) in a turbulent jet. Note that in the current LES $d / h=0.103$ and $y_{s_{a v}} / h=0.1185$ are smaller than those considered in the experiments of Costa-Patry and Mydlarski (2008) and therefore quantitative comparison is not made. However, if the two sources are separated by a small distance, e.g. $d / h \leq 0.13$, qualitative comparison with Costa-Patry and Mydlarski (2008) and Vrieling and Nieuwstadt (2003) shows that the correlation coefficient initially is negative and then gradually becomes positive with increasing downstream distance. In the near field, the plume widths are small and the transport of the plumes by the same eddies yield anti-phase contributions to the correlation. In contrast, the positive correlation is a result of the two plumes tending to pass the measurement location simultaneously. Or as aptly described by Costa-Patry and Mydlarski (2008), the plume flapping in the near field and internal turbulent mixing in the far field determines the sign of the cross correlation. Note that for $x / h<1$, the plumes are narrow and hence the cross 
correlation at the plume edges (i.e. at $y / h=0.0182$ and 0.2231 ) are subjected to numerical discrepancies. All the profiles approach asymptotic values between 0.7 to 0.9 at very large $x / h$, indicating that the source heights have little effect on dispersion in the far field.

To understand the development of the relative intensity of the concentration fluctuations with downstream distance, the maximum concentration fluctuation $c_{r m s-m a x}$ normalised by the maximum mean concentration $\bar{c}_{\max }$ is plotted in Fig. 17 for the three line sources. The locations of the peak in the $c_{r m s-\max } / \bar{c}_{\max }$ variation for the near wall sources are closer to the sources than that for the centre line source, which is consistent with the results of dispersion from point sources (Fackrell and Robins, 1982; Xie et al., 2004). This is due to the difference in the local turbulence intensity, the local turbulence integral length scale and the impedance effect of the wall. For $y_{s} / h=0.067$, the fall from the peak value is the most rapid. From approximately $x / h=2.0$ (where the maximum mean concentration is at the wall), $c_{r m s-\max } / \bar{c}_{\max }$ falls only very slowly. In contrast, for $y_{s} / h=0.17$, the downstream fall from the peak is not as rapid as that for $y_{s} / h=0.067$. However, from approximately $x / h=6.0$ (where the maximum mean concentration is at the wall), the $c_{r \text { ms }-\max } / \bar{c}_{\text {max }}$ decay is very slow and almost coincides with the variation for $y_{s} / h=0.067$. This suggests that the effect of the height of the near wall source on the relative concentration fluctuation is negligible downstream of $x / h=6.0$. In the experiments, however, the difference between the relative intensity variations for the two near wall sources is visible upstream of $x / h=$ 15 (not shown here). Downstream of $x / h=15$, the two variations seemed to approach a constant of about 0.3. All these observations might seem consistent with the speculation that an asymptotic non-zero value of $c_{r \operatorname{ms}-\max } / \bar{c}_{\max }$ in dispersion from point sources exists (see Xie et al., 2007) (but see below, where we argue that this cannot be true in channel flows when extremely long domains are considered). The peak of $c_{r m s-\max } / \bar{c}_{\max }$ for $y_{s} / h=0.067$ is less than that for $y_{s} / h=0.17$, which is probably because of the smaller turbulence integral length scale and stronger wall impedance effect in the former compared with the latter.

For the centreline source, the $c_{r \operatorname{ms}-\max } / \bar{c}_{\max }$ behaviour is quite different from that for the near wall sources. In the near wall region the turbulence intensity is much higher (Fig. 5) and the transverse turbulence integral scales are smaller than those in the centre of the channel (Xie et al., 2004; Iwamoto, 2002). So for near wall sources, due to stronger turbulent convection, the concentration fluctuation is quickly enhanced and $c_{r m s-\max } / \bar{c}_{\max }$ rapidly reaches its peak. Then the scalar approaches a well-mixed state more quickly due to the shorter local integral scale. On the other hand, for the centre line source, the concentration fluctuation increases relatively slowly due to the shorter mixing time caused by higher velocity at the channel centre and weaker local turbulence intensity. Then the plume flapping enhances the concentration fluctuation significantly in the far field and hence the peak in $c_{r \operatorname{ms}-\max } / \bar{c}_{\text {max }}$ occurs further downstream (beyond $x / h>4$ ) than for near wall source cases.

It should be noted that once the scalar has become fully mixed across the entire channel, which must eventually happen wherever the source is placed, the mean concentration will be uniform for all $y$ and independent of source location (provided the volume flux is the same). It would then not be possible to deduce the source location (in $y$ ). On the other hand, perhaps unlike the boundary layer case of Xie et al. (2007), the r.m.s. of the scalar fluctuation must eventually decay to zero in a channel flow, due to the dissipation term in the variance transport equation, since there is no mechanism to maintain the fluctuations (because the mean scalar concentration gradient is zero). The data in Fig. 17 confirm the expectations that, firstly, this eventual decay in $c_{r m s-\max } / \bar{c}_{\text {max }}$ to zero is very slow and, secondly, that it will take much longer for a source near the centre-line than for one near the wall.

Because of the high uncertainty in the measurement of mean scalar in the experiments (LM), the experimental data $c_{r \operatorname{ms}-\max } / \bar{c}_{\max }$ should only be interpreted qualitatively as sug- 
gested by LM. For the centre-line source, the peak of the relative intensity of scalar fluctuations in the experiments was found to be 1.75 (at $x / h=4$ ) and gradually decreased to 0.8 (at $x / h=18.6$ ). The current LES shows the peak to be approximately 0.6 at $x / h \approx 4$ (for the rectangular source it is slightly lower), decreasing monotonically to 0.5 at $x / h \approx 11$. We found from further numerical experiment that turning off the sub-grid scale model showed negligible difference to the relative intensity of concentration fluctuations. This suggests that the sub-grid scale Schmidt number effects are not significant for the resolution used. Lower relative intensity of scalar fluctuations in the computations are therefore partly attributed to the much larger source size. Fackrell and Robins (1982) showed that the effect of source size on $c_{r m s-\max } / \bar{c}_{\max }$ can be significant, although their experiments showed that it was negligible for ground level sources. Numerical experiments for investigating the effect of source size were performed (Table 3). For the near wall sources with small size (Set 1), there is about a $10 \%$ increase in the peak (Fig. 17). Note the small and large source sizes for near wall sources are $3 \eta$ and $5 \eta$ respectively. Such a range of sizes is clearly insufficient for investigating the entire range of possible source size effects but the data show that downstream of the peak the effect of source size in the present cases vanishes rapidly. For the centre line source, the difference in the results between Set 1 and Set 2 is very small, which is probably because the effective size of the rectangular source in Set 1 is approximately equivalent to that of the Gaussian source in Set 2. With regards to any comparison of dispersion from point sources and line sources, the source size effects are expected to be smaller for the latter. Also, because of the homogeneity of the line source dispersion in the spanwise direction, intermittency of the scalar is expected to be weaker than that for point sources. Consequently, both $c_{r \operatorname{ms}-\max } / \bar{c}_{\max }$ and measures of the relative extreme concentrations (i.e. maximum possible concentration normalised by the mean, Xie et al., 2007; Mole et al., 2008) are expected to be lower for line than for point sources.

In the DNS study of Vrieling and Nieuwstadt (2003) at $R e_{\tau}=180$, the peak $c_{r m s-\max } / \bar{c}_{\text {max }}$ (for the central line source) was found to be 0.8 at $x / h \approx 4$. This is not inconsistent with our LES data, given the possible effects of source size and $R e$ number. Much smaller values were observed by Karnik and Tavoularis (1989) in uniform shear turbulence. The relative intensity of the scalar fluctuations was approximately 0.5 at $x / M \approx 6$ and far downstream $(x / M \approx 120)$ it had decreased to 0.15 . Viswanathan and Pope (2008) estimated the asymptotic value of the relative intensity to be 0.4 in their grid turbulence experiments. Collectively, all these results suggest that the asymptotic value of $c_{r m s-\max } / \bar{c}_{\max }$ is very dependent on the specific type of flow, although we emphasise that in channel (or pipe) flows it must be zero.

\subsubsection{Probability-density functions}

The probability density functions of the normalised scalar fluctuations at five downstream locations and at their respective source heights are shown in Fig. 18 for all three line sources. In the near field i.e. at $x_{c} / h=1$ and 2.5 , all three line sources exhibit different shapes of pdf due to different turbulent intensities and integral length scales. The pdf profiles from the near wall source show 'Gaussian-like' shapes and those from the centre line source are negatively skewed because of large scale plume meandering. The pdf profile from the source at $y_{s} / h=$ 0.17 shows a more 'uniform' distribution, but with longer positive tails. Further downstream, both the near wall source pdfs tend towards a Gaussian shape and are also found to be in good agreement with experiments. Unlike LES, the pdfs from the centre line source are spiky and positively skewed in the experiments. Bakosi et al. (2007) also observed similar profiles at $R e_{\tau}=1080$ for the centre line source. But their pdfs from the near wall source 


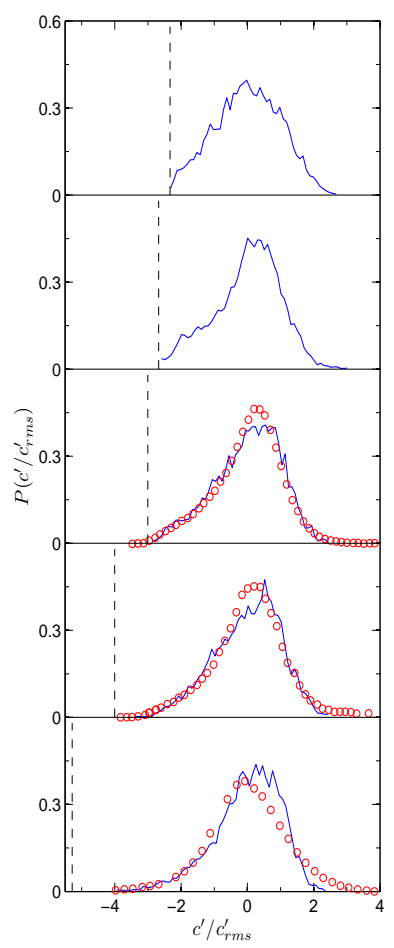

(a) $y_{s} / h=0.067$

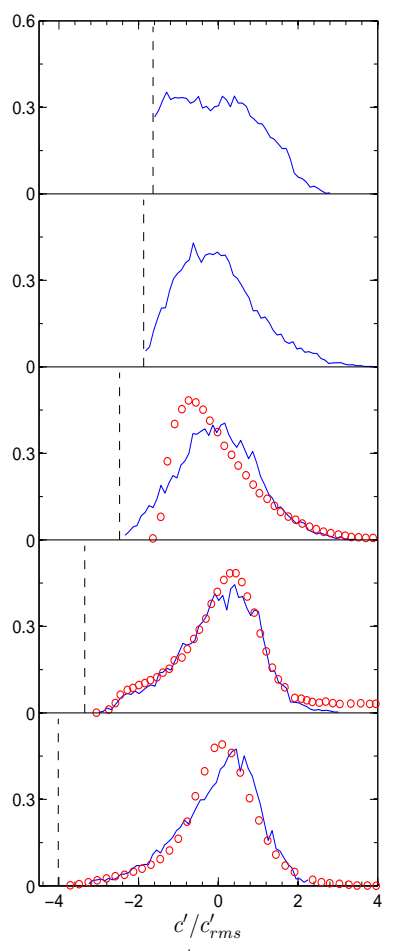

(b) $y_{s} / h=0.17$

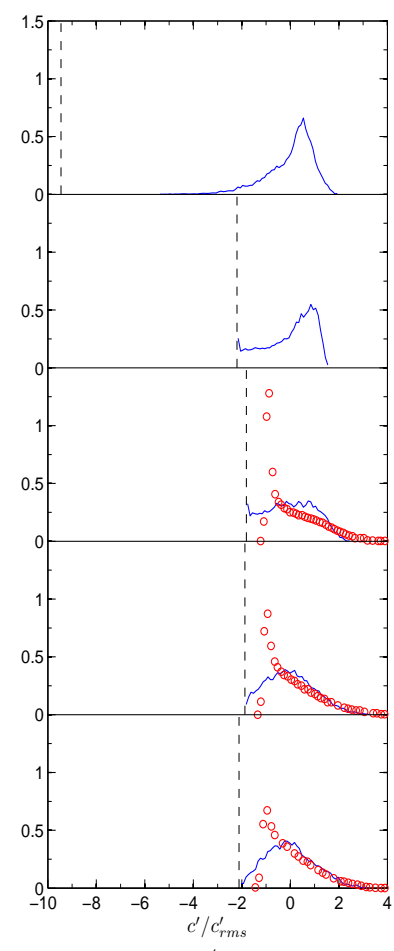

(c) $y_{s} / h=1$

Fig. 18 PDFs of the scalar fluctuations at the plume centreline and at different downstream distances. Row 1 corresponds to $x_{c} / h=1$, Row 2: $x_{c} / h=2.5$, Row 3: $x_{c} / h=4$, Row 4: $x_{c} / h=7.4$ and Row 5: $x_{c} / h=10.8$. The vertical dashed line in the graphs correspond to scalar concentration, $c=0$ in LES. Lines: LES, symbols: experiments.
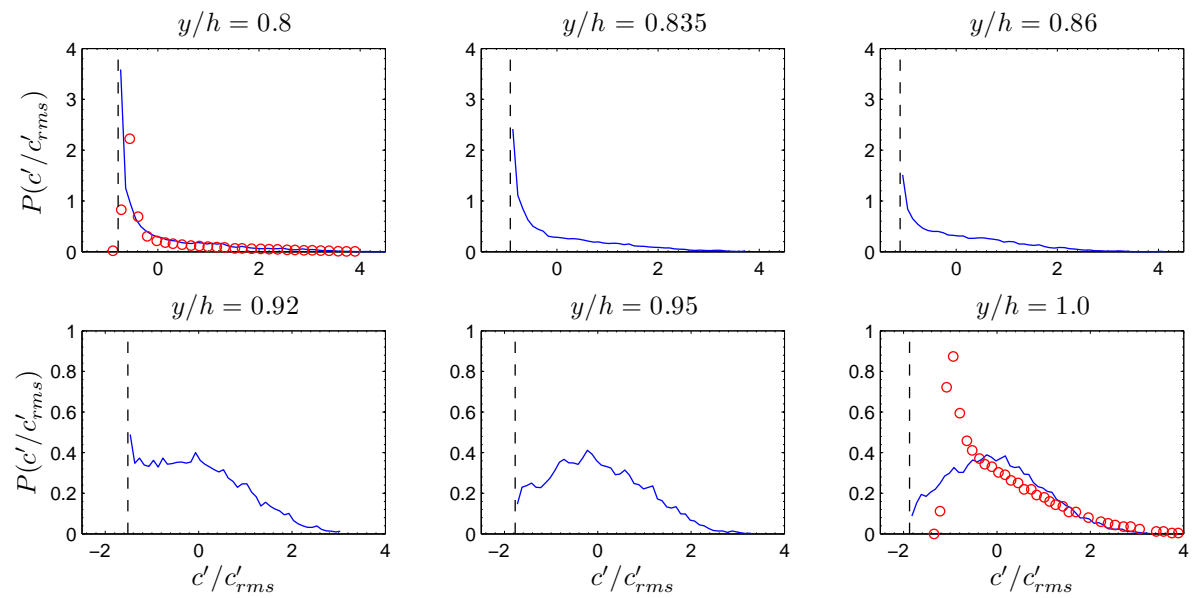

Fig. $19 \mathrm{PDF}$ of the scalar fluctuations for centre line source at $x_{c} / h=7.4$ and at different wall normal locations. The vertical dashed line corresponds to scalar concentration, $c=0$ in LES. Lines: LES, symbols: experiments. 


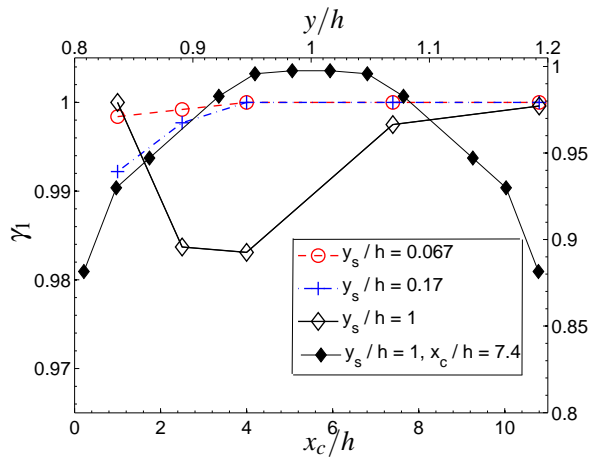

(a) $\gamma_{1}=\mathrm{P}(c>0.02 \bar{c})$

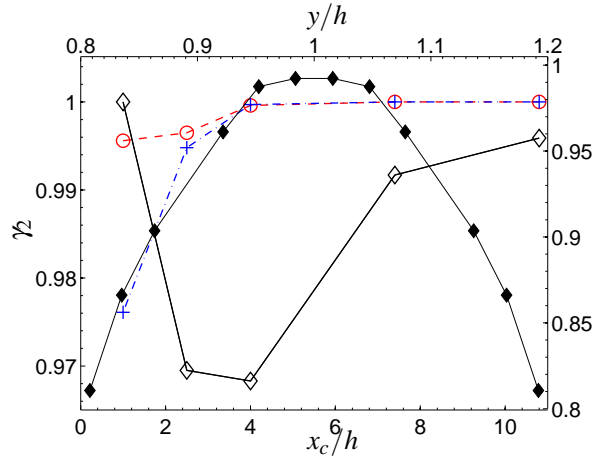

(b) $\gamma_{2}=\mathrm{P}(c>0.05 \bar{c})$

Fig. 20 Variation of intermittency factor at the plume centreline with the downstream distance for the three line sources. Intermittency factor is also shown across the plume at $x_{c} / h=7.4$ for the centre line source (for clarity, its $y$-axis is labelled on the right side of the figure). Legend in (b) is same as in (a)

at $y_{s} / h=0.067$ are also spiky and negatively skewed, unlike the Gaussian shape seen in experiments, and they attributed this to the inaccurate specification of the micromixing time scale in their model. We are not entirely sure if this is the reason for their spiky skewed pdf even for the centre line source. The integral length scales and plume widths are almost of the same order at far downstream locations from the source. In experiments, the source size being smaller, the scalar dispersion is due to the result of the plume flapping as well as the turbulent advection. As a result, the scalar fluctuating intensities are also high in experiments. Whereas in LES, the source size being bigger and transverse fluctuations being under-resolved, the effects of plume flapping are relatively weaker. Hence, pdfs are not spiky in LES. However, it can be argued that the spiky pdf in experiments is not due to the bulk flapping of the plume but could result from the measurements taken slightly away from the channel centre line. Note in Fig. 10c that the mean concentration profiles of experiments are not symmetric with respect to the channel centre at $x_{c} / h=7.4$. Figure 19 shows the corresponding LES pdfs of the scalar fluctuations from the centre line source at different transverse locations. It can be observed that even at small distances from the channel centre, pdf profiles are very different. Near the plume edges, pdfs show an exponential decay for high scalar fluctuations due to the intermittency.

The intermittency factor is generally defined as the probability that the scalar concentration is non-zero, i.e. $\gamma=\mathrm{P}(c>0)$. We observed that the scalar concentration is always non-zero at several downstream locations for all the three line sources, except in the very near field. Therefore, intermittency factor is defined here using a threshold and so results depend on the chosen threshold value. Sykes and Henn (1992) chose the threshold to be 5\% of the mean concentration value based on their grid studies. We define $\gamma_{1}=\mathrm{P}(c>0.02 \bar{c})$ and $\gamma_{2}=\mathrm{P}(c>0.05 \bar{c})$ and scalar intermittency for these two cases are shown in Figs. 20a and $20 \mathrm{~b}$ for different downstream locations and also across the plume from the centre line source at $x_{c} / h=7.4$. It is to be noted that the profiles are similar except that $\gamma_{1}$ showed higher intermittency compared to $\gamma_{2}$ as expected. Figure $20 \mathrm{~b}$ shows that for the two near wall sources, intermittency is lower near the source and gradually asymptotes to one for $x_{c} / h>4$. The plume from $y_{s} / h=0.17$ exhibited lower intermittency near the source compared with that from $y_{s} / h=0.067$. This is expected because the latter is subjected to shorter integral length scales because of the presence of the bottom wall of the channel. For the centre line source, 


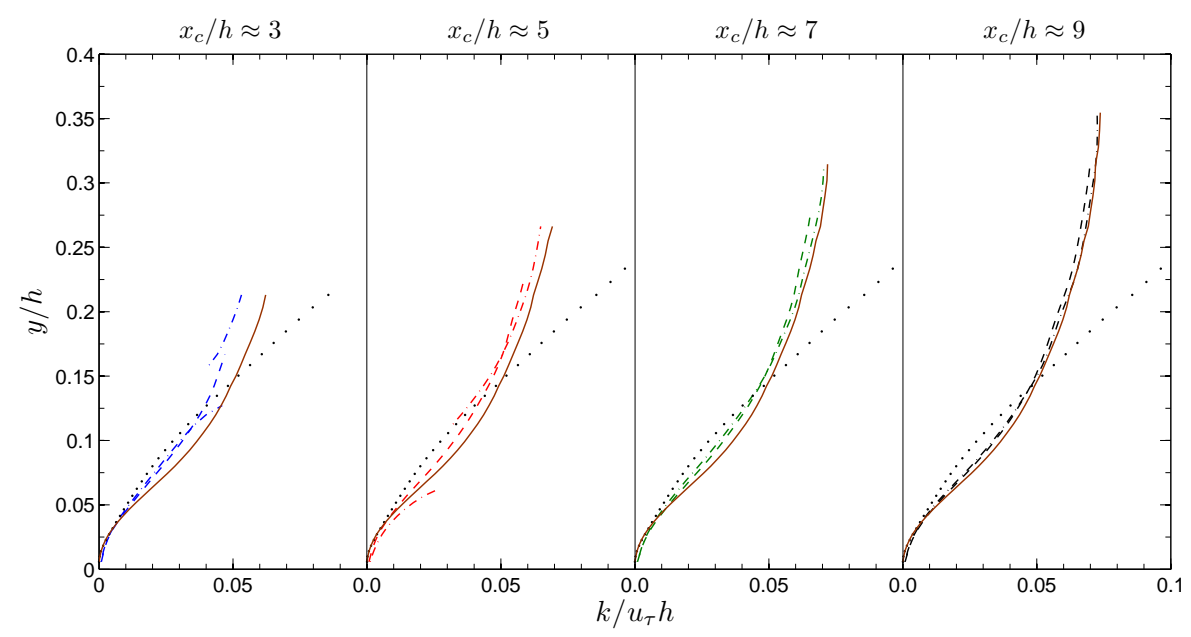

Fig. 21 Vertical eddy diffusivity for the two near wall sources. Dashed line: $y_{s} / h=0.067$, LES; dot-dashed line: $y_{s} / h=0.17$, LES; solid line: DNS; dotted line: mixing length model.

the intermittency factor exhibits non-monotonic behavior. Near the source, the intermittency factor is high and this can be attributed to the large source size and lower turbulence intensity at the channel centre. The source size effects on the intermittency are studied in detail by Fackrell and Robins (1982) in a turbulent boundary layer. Their elevated point source of larger size shows similar intermittency behavior to that found in the present case of centre line source as expected. Away from the source, the intermittency factor decreases but then increases with increase in downstream distance. This is because the plume is subjected to meandering in the initial stages before it becomes more diffusive across. As a result, lower intermittency is observed at the plume centreline. But far downstream end, the plume size increases resulting in intermittency closer to unity. Compared to the point source in a turbulent boundary layer (Fackrell and Robins, 1982), the intermittency for a line source is observed to be larger because the former plume is subjected to meandering in the spanwise direction also. The intermittency across the plume from the centre line source is also shown in Fig. 20b. As expected, the values of $\gamma_{2}$ are larger near the plume axis and smaller at the plume edges.

\subsubsection{Eddy diffusivity}

Livescu et al. (2000) showed that the diffusion coefficient from the $k-\varepsilon$ model differed from that derived by DNS in the turbulent convective and transition to turbulent diffusive regimes of scalar dispersion in grid turbulence. However, Karnik and Tavoularis (1989) found some clues on the validity of gradient transport concept for scalar dispersion in the diffusive regime in uniform shear flows. We now consider this further.

The vertical eddy diffusivity, $k$ is obtained from the gradient transport relation

$$
\overline{c^{\prime} v^{\prime}}=-k \frac{d \bar{C}}{d y} .
$$

At four downstream locations for the two near wall sources, normalised diffusivity values are shown in Fig. 21 for $y \leq 2 \sigma$. The discontinuity observed in some of the LES profiles 
in Fig. 21 is near the region where the mean concentration gradient is zero. The diffusivity values from Eq. 5 are compared with those estimated from the DNS data (Moser et al., 1999) using the equation

$$
k=v_{t} / S c_{t},
$$

where $v_{t}$ is the turbulent eddy viscosity and $S c_{t}$ is turbulent Schmidt number, which is assumed here as unity. Also shown in Fig. 21 is the diffusivity estimated from the standard mixing length model of the turbulent boundary layer, given by

$$
v_{t}=l_{m}^{2}\left|\frac{d U}{d y}\right|, \quad \text { where } \quad l_{m}=\kappa y\left[1-\exp \left(-y^{+} / 26\right)\right]
$$

and $\kappa=0.4$. It is observed that for the two near wall sources the diffusivity obtained from Eq. 5 is almost the same. The diffusivity changes with the downstream distance slightly, indicating a small variation in the turbulent Schmidt number. The LES estimates from Eq. 5 are found to be in good agreement with DNS (Eq. 6) in the far field (e.g. $x_{c} / h \approx 9$ ), suggesting that (i) a constant turbulent Schmidt number is a reasonable assumption in the far field; and (ii) the gradient transport concept for dispersion might be valid provided the turbulent viscosity is known. These diffusivity values are in fair agreement with those obtained from the mixing length model (Eq. 7 ) up to $y / h \approx 0.15$ or $y^{+} \approx 80$, which suggests that the gradient transport concept can be used at least qualitatively to predict the dispersion in these regimes.

For elevated point source dispersion in a rough wall turbulent boundary layer, Fackrell and Robins (1982) obtained approximately constant diffusivity in the very near field, but this started to deviate towards profiles somewhat similar to those shown in Fig. 21 in the far field. They observed an increase in $k$ with increasing downstream distance and the values are found to be slightly greater than those in the current study. Again these observations suggest that the gradient transport model might be useful to estimate vertical dispersion in the near wall regions for the near wall sources. In the case of the centre line source, the plume widths are much lower and integral length scales are greater than those for the two near wall sources. Therefore, it is difficult to comment on the validity of the gradient transport concept for the centre line source.

\section{Conclusions and discussions}

Firstly, large-eddy simulation (LES) implemented in Star-CD v4.06 was performed for passive scalar dispersion from a line source in a plane channel flow at $R e_{\tau}=180$. The results are in encouraging agreement with the DNS data in the literature. Then passive scalar dispersion from three line sources in a fully developed turbulent channel flow at $R e_{\tau}=520$ was studied using LES, with line sources placed at $y_{s} / h=0.067,0.17$ and 1.0, corresponding to $y_{s}^{+}=35,87,520$ respectively. The resulting mean and fluctuating quantities of the scalar were then compared with the experiments (LM). For the three sources, the LES predicted mean plume width is slightly less than that from the experiments. Given the uncertainties in measuring the mean scalar due to the inaccuracy in estimating the free-stream temperature in the experiments and slightly under-resolved transverse fluctuations in LES, perfect agreement between the LES and the experiments could not be expected for the mean scalar field. For the sources at $y_{s} / h=0.067$ and 0.17 , LES showed the peak of the mean concentration to be at the bottom wall from approximately $x / h>2$ and 6 respectively, as expected. In the experiments, however, for the downstream locations considered, the peak of the mean scalar shifting towards the wall occurs very gradually. 
The comparison of the vertical $c_{r m s}$ profiles between the LES and experiments is better than that of the mean scalar profiles, apart from the discrepancy of the location of the peak $c_{r m s}$ (which is probably due to the discrepancy of the location of the peak mean). This may confirm the comment in LM that the fluctuating temperature field is not subject to the effects of room temperature variations and is therefore significantly more precise. Both the LES and experiments showed that the peaks of the $c_{r m s}$ from the near wall sources drift away from the bottom wall, whereas the peak of the latter is further away from the wall than that of the former. For the centre line source, double peaks are observed in the r.m.s profiles of the scalar fluctuation at far downstream locations, e.g. $x / h \geq 7.4$. Such double peaks were absent in the experiments, probably because the observed small ripple of the double peak is within the experimental error.

The effect of source size (with $\sigma_{s}^{+}$varying from 3 to 5) on the mean concentration $\bar{c}$ is found negligible in the far field, e.g. $x / h \geq 3$. The difference of the relative intensity of concentration fluctuation $c_{r \operatorname{ms}-\max } / \bar{c}_{\max }$ from the two sets of sources is no more than $10 \%$. Downstream of the location where the peak of the profile $c_{r m s-\max } / \bar{c}_{\text {max }}$ occurs, this difference vanishes quickly. The peak of $c_{r \operatorname{rms}-\max } / \bar{c}_{\max }$ for the centre line source in the experiments is found to be more than twice that in the LES data, which is mainly due to the much smaller source size used in the experiments.

LES results suggest that the effect of the height of the near wall sources, i.e. $y_{s} / h=$ 0.067 and 0.17 , on the relative intensity of concentration fluctuations is negligible downstream of $x / h=6.0$. This is not surprising because the plume is likely to be well mixed at least throughout the near wall region in the far field and the effect of source height thus disappears. Whilst the LES data might also suggest that the relative scalar fluctuation intensity approaches a non-zero constant in the far field, as in boundary layers (Xie et al., 2007), it must in fact eventually decay to a zero value in a channel flow. This requires very long downstream distances, particularly for sources nearer the channel centre-line.

The pdfs of the scalar fluctuations from the two near wall sources are found to be in fair agreement with the experiments. For the center line source, the pdfs are found to be spiky and positively skewed in experiments, but not in LES and this could be because of the larger source size and under-resolved transverse fluctuations. However uncertainties in the location of the measurement probe in experiments also could result in such spiky behavior as LES showed that away from the channel center line, the pdfs are quite different. Fair agreement of eddy diffusivities from mixing length model and gradient transport concept suggests that the latter can be at least used qualitatively to predict the dispersion in the near wall regions.

Whilst this study has shown that LES is a promising tool for understanding scalar dispersion in shear flows, more rigorous studies will be needed before extension to dispersion in very high Reynolds number flows (as in urban environments) can be successfully implemented.

Acknowledgements This project is funded by the Natural Environment Research Council, through its National Centre for Atmospheric Sciences, Grant No. R8/H12/38. We thank Dr Laurent Mydlarski for providing the experimental data and helpful discussions. We thank the referees for their valuable comments. All computations have been done using CD-adapco's STAR-CD code on Iridis (University's supercomputer) and HECToR (U.K's supercomputer) and the authors are grateful to Dr Fred Mendonca for his continuous support. 


\section{References}

Anand M, Pope SB (1985) Diffusion behind a line source in grid turbulence. In: Bradbury LJS, Durst F, Launder BE, Schmidt FW, Whitelaw JH (eds) Turbulent Shear Flows 4, Springer - Verlag, pp 46-61

Bakosi J, Franzese P, Boybeyi Z (2007) Probability density function modeling of scalar mixing from concentrated sources in turbulent channel flow. Phys Fluids 19:115,106, DOI 10.1063/1.2803348

Bernard PS, Rovelstad AL (1994) On the physical accuracy of scalar transport modeling in inhomogenous turbulence. Phys Fluids 6(9):3093-3108

Boppana VBL, Xie ZT, Castro IP (2010) Large-eddy simulation of dispersion from surface sources in arrays of obstacles. Boundary Layer Meteorol 135(3):433-454

Brethouwer G, Nieuwstadt FTM (2001) DNS of mixing and reaction of two species in a turbulent channel flow: a validation of the conditional moment closure. Flow Turbulence Combust 66:209-239

Brethouwer G, Boersma BJ, Pourquié MBJM, Nieuwstadt FTM (1999) Direct numerical simulation of turbulent mixing of a passive scalar in pipe flow. Eur J Mech B / Fluids 18:739-756

Brown RJ, Bilger RW (1996) An experimental study of a reactive plume in grid turbulence. J Fluid Mech 312:373-407

Cho MS, Chung MK (1997) Application of a reynolds stress / heat flux model to the turbulent thermal dispersion behind a line heat source in a uniformly sheared flow. Numerical Heat Transfer, Part A: Applications 32(7):715-732

Costa-Patry E, Mydlarski L (2008) Mixing of two thermal fields emitted from line sources in turbulent channel flow. J Fluid Mech 609:349-375

Fabregat A, Pallarès J, Cuesta I, Grau FX (2009) Dispersion of a buoyant plume in a turbulent pressure-driven channel flow. Int J Heat and Mass Transfer 52:1827-1842

Fackrell JE, Robins AG (1982) Concentration fluctuations and fluxes in plumes from point sources in a turbulent boundary layer. J Fluid Mech 117:1-26

Iliopoulos I, Hanratty TJ (1999) Turbulent dispersion in a non-homogenous field. J Fluid Mech 392:45-71

Iwamoto K (2002) Database for fully developed channel flow. THTLAB Internal Report (ILR-0201), Dept Mech Eng, Univ Tokyo DNS database (CH12_PGWL7) http://www.thtlab.t.u-tokyo.ac.jp/

Karnik UA, Tavoularis S (1989) Measurements of heat diffusion from a continuous line source in a uniformly sheared turbulent flow. J Fluid Mech 202:233-261

Kim J, Moin P, Moser RD (1987) Turbulence statistics in fully developed channel flow at low reynolds number. J Fluid Mech 177:133-166

Kyong NH, Chung MK (1987) Turbulent scalar transport correlation behind a line heat source in a uniform shear flow. Korean Society of Mechanical Engineers Journal 1(2):95100

Lavertu RA, Mydlarski L (2005) Scalar mixing from a concentrated source in turbulent channel flow. J Fluid Mech 528:135-172

Liu CH, Barth MC (2002) Large-eddy simulation of flow and scalar transport in a modeled street canyon. J App Meteorol 41:660-673

Livescu D, Jaberi FA, Madnia CK (2000) Passive-scalar wake behind a line source in grid turbulence. J Fluid Mech 416:117-149

Mole N, Schopflocher TP, Sullivan PJ (2008) High concentrations of a passive scalar in turbulent dispersion. J Fluid Mech 604:447C474 
Moser RD, Kim J, Mansour NN (1999) Direct numerical simulation of turbulent channel flow up to $R e=590$. Phys Fluids 11(4):943-945

Shah KB (1998) Large eddy simulations of flow past a cubic obstacle. Dissertation Stanford University

Shraiman BI, Siggia ED (2000) Scalar turbulence. Nature 405:639-646

Sreenivasan KR (1991) On local isotropy of passive scalars in turbulent shear flows. Proc R Soc London Ser A 434:165-182

STAR-CD (2009) CD-adapco's CCM user guide, STAR-CD Version 4.08

Sykes RI, Henn DS (1992) Large-eddy simulation of concentration fluctuations in a dispersing plume. Atmos Environ 26A(17):3127-3144

Thomson DJ (1990) A stochastic model for the motion of particle pairs in isotropic highreynolds-number turbulence, and its application to the problem of concentration variance. J Fluid Mech 210:113-153

Tong C, Warhaft Z (1995) Passive scalar dispersion and mixing in a turbulent jet. J Fluid Mech 292:1-38

Townsend AA (1954) The diffusion behind a line source in homogenous turbulence. Proc R Soc London Ser A 224:487-512

Viswanathan S, Pope SB (2008) Turbulent dispersion from line sources in grid turbulence. Phys Fluids 20:101,514, DOI 10.1063/1.3006069

Vrieling AJ, Nieuwstadt FTM (2003) Turbulent dispersion from nearby point sourcesinterference of the concentration statistics. Atmos Env 37:4493-4506

Walton A, Cheng AYS (2002) Large-eddy simulation of pollution dispersion in an urban street canyon - part II: idealised canyon simulation. Atmos Environ 36:3615-3627

Wang Y, Komori S (1999) Application of a second-moment closure model to simulate the turbulent dispersion from an elevated line source. Heat and Mass Transfer 34:429-436

Warhaft Z (1984) The interference of thermal fields from line sources in grid turbulence. J Fluid Mech 144:363-387

Warhaft Z (2000) Passive scalars in turbulent flows. Annu Rev Fluid Mech 32:203-240

Xie ZT, Castro IP (2008) LES and RANS for turbulent flow over arrays of wall-mounted cubes. Flow, Turb \& Comb 76:291-312

Xie ZT, Castro IP (2009) Large-eddy simulation for flow and dispersion in urban streets. Atmos Environ 43(13):2174-2185

Xie ZT, Hayden P, Voke PR, Robins AG (2004) Large-eddy simulation of dispersion: comparison between elevated and ground-level sources. J Turbulence 5(31):1-16

Xie ZT, Hayden P, Robins AG, Voke PR (2007) Modelling extreme concentration from a source in a turbulent flow over rough wall. Atmospheric Environment 41(16):3395-3406 